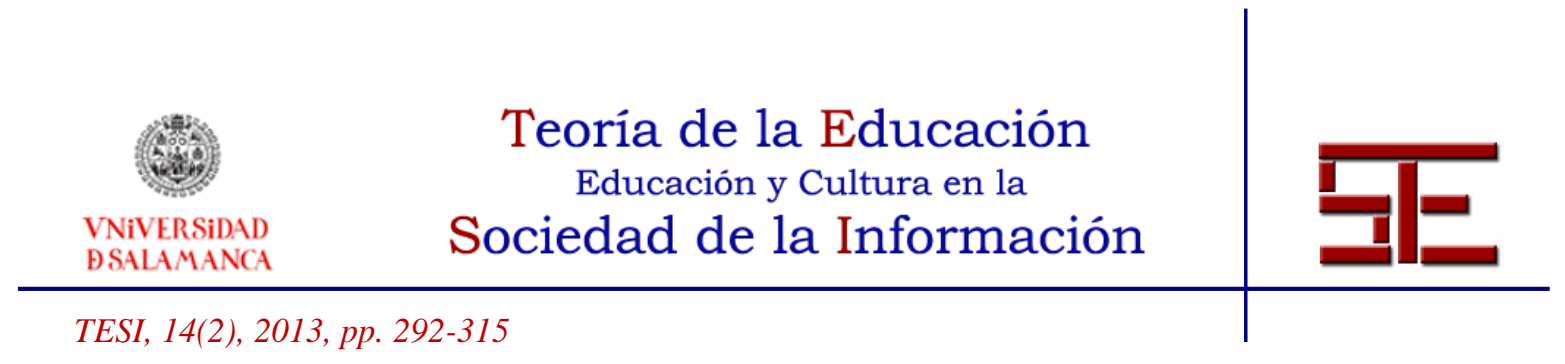

\title{
LA PLANIFICACIÓN ESTRATÉGICA, UN INDICADOR SOBRE EL LIDERAZGO PEDAGÓGICO
}

Resumen: Destacando la función del liderazgo pedagógico como factor decisivo para la eficacia en el funcionamiento de los centros educativos, este artículo analiza la planificación estratégica como indicio de la presencia de dicho factor crítico en la dinámica de las escuelas e institutos españoles. En el análisis de las variables asociadas al nivel de desarrollo de este tipo de actividad directiva, se pone de manifiesto su vinculación con el liderazgo para el aprendizaje al que se concede una mayor potencialidad por su contribución al éxito educativo. El estudio de los indicios disponibles sobre el nivel de desarrollo alcanzado por esta forma de acción estratégica pone de manifiesto su vinculación a las creencias de los directores más que a las condiciones que establece el contexto en que se circunscribe la actividad de las escuelas. Los resultados se han obtenido de una muestra de 694 directores, representativos de los centros educativos españoles en sus etapas obligatorias.

Palabras clave: Liderazgo pedagógico; liderazgo instruccional; liderazgo transformacional; eficacia escolar; mejora escolar; reestructuración escolar; planificación estratégica; dirección de centros educativos.

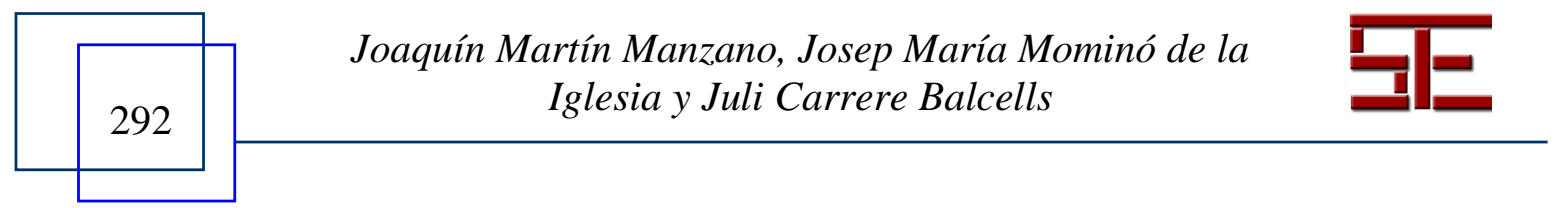




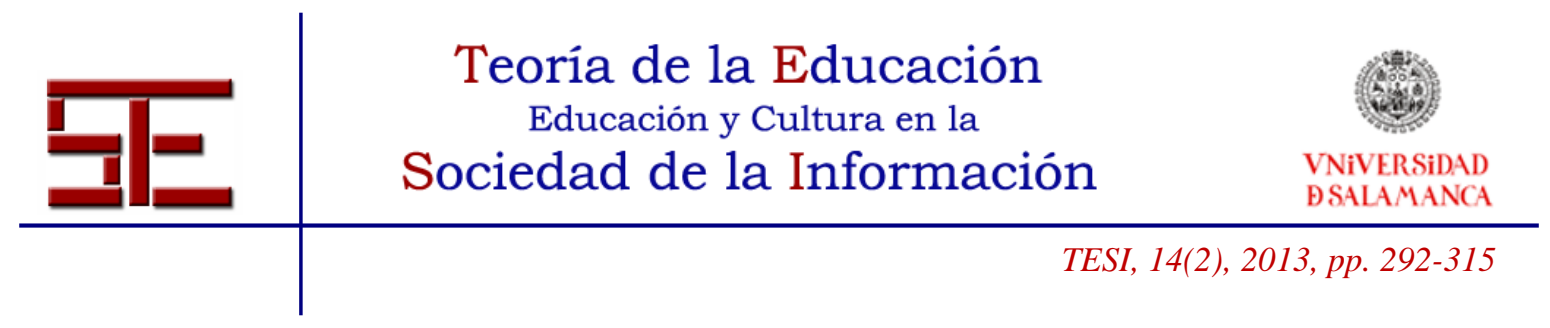

\title{
THE STRATEGIC PLANNING, AN EDUCATIONAL LEADERSHIP INDICATOR
}

\begin{abstract}
Emphasizing the role of the pedagogical leadership as a decisive factor for the efficient operation of the educational centers, this article discusses the strategic planning as an indication of the presence of this critical factor in the dynamics of the schools and institutes in Spain. In the analysis of the variables associated with the level of development of this type of directive activity, it reveals its links with the leadership for learning that are given greater potential to its contribution to the educational success. The study of the available evidence on the level of development reached by this form of strategic action underscored its commitment to the beliefs of directors rather than to the conditions set out the context which restricted the activities of the schools. The results have been obtained from a sample of 694 directors, representative of the Spanish school, in their mandatory stages.
\end{abstract}

Key words: Educational leadership; instructional leadership; transformational leadership; school effectiveness; school improvement; school restructuring; strategic planning; school direction.

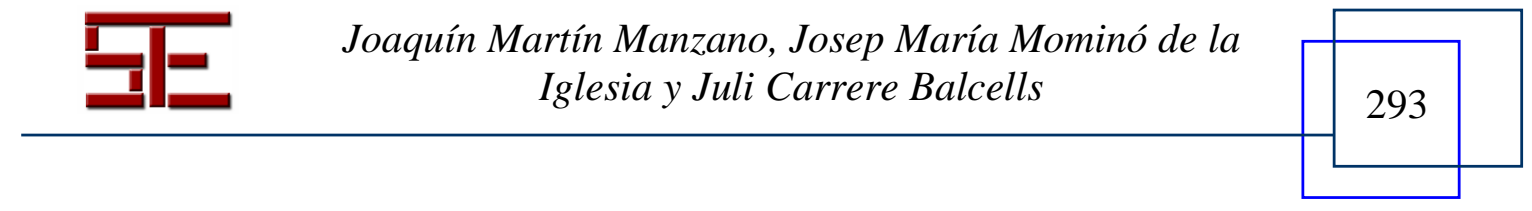




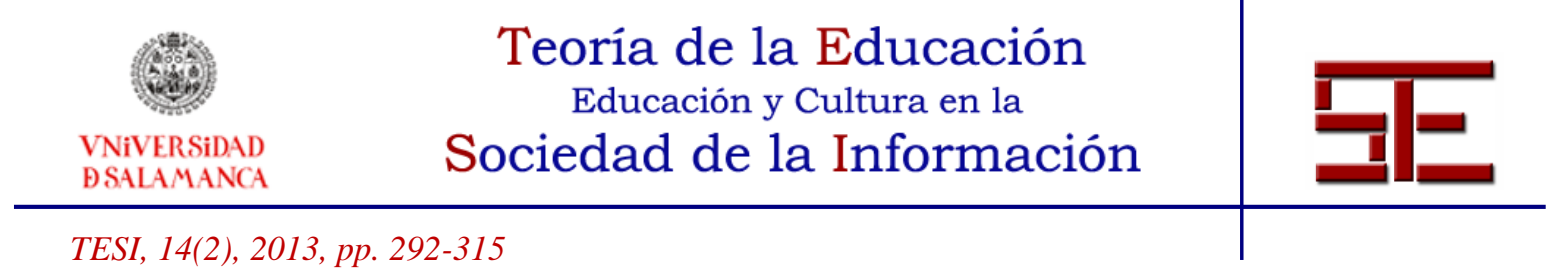

\section{LA PLANIFICACIÓN ESTRATÉGICA, UN INDICADOR SOBRE EL LIDERAZGO PEDAGÓGICO}

Fecha de recepción: 20/03/2013; fecha de aceptación: 20/05/2013; fecha de publicación: 30/07/2013

Joaquín Martín Manzano

niuqao@gmail.com

Universitat Oberta de Catalunya

Josep Maria Mominó de la Iglesia

jmomino@uoc.edu

Universitat Oberta de Catalunya

Juli Carrere Balcells

jcarrere@uoc.edu

Internet Interdisciplinary Institute (IN3)

\section{1.- INTRODUCCIÓN}

De entre las variables que afectan al hecho educativo que configuran y modelan un centro educativo, el artículo se centra en el liderazgo pedagógico y, más específicamente, en la planificación estratégica como indicio de la presencia de este tipo de liderazgo. Este indicador se considera fundamental puesto que el liderazgo no es observable a simple vista, sino que destila de las acciones, de unas estrategias, que evidentemente persiguen la consecución de unos objetivos a corto, medio o largo plazo.

El modelo técnico-directivo de dirección escolar, en España ha tenido una presencia fundamental que alcanza prácticamente hasta nuestros días, aunque de forma relativamente incipiente se puede observar el énfasis en la idea de liderazgo pedagógico, vinculada al movimiento de las escuelas eficaces (Reynolds, 1976; Reynolds y Creemers, 1990, Murphy et al., 2006). Esta forma de liderazgo educativo, dirigida prioritariamente a la mejora de los procesos de aprendizaje, se observa como un factor fundamental para la mejora del rendimiento académico, objetivo fundamental de los centros educativos. ¿Existen indicios de este cambio de perspectiva en la acción directiva? ¿En qué medida los directores y directoras de las escuelas e institutos han empezado a actuar como líderes pedagógicos? Una aproximación, a partir de un análisis empírico, puede contribuir a comprender cuál es el nivel alcanzado en este proceso y a identificar la naturaleza de algunos de los factores implicados en su desarrollo. Para este propósito, de entre todos

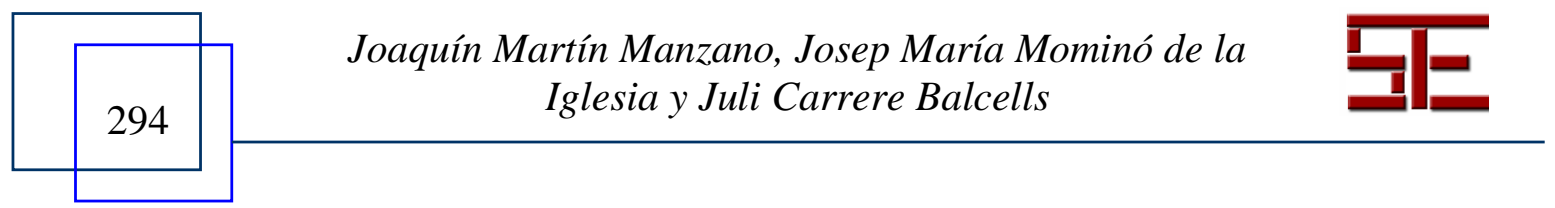




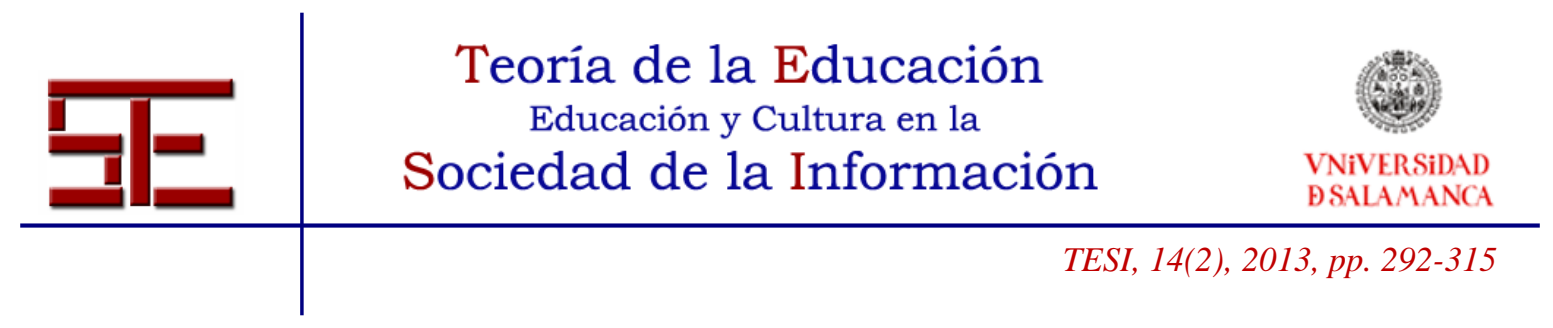

los indicios de liderazgo, este artículo pone la atención en la planificación estratégica y, específicamente, en su orientación al aprendizaje como rasgo distintivo del liderazgo pedagógico.

\subsection{El liderazgo pedagógico, un factor clave para la mejora de los centros educativos}

La investigación sobre la eficacia escolar ha puesto de manifiesto, en el transcurso de las últimas décadas, el papel destacado del liderazgo como factor asociado a la calidad de los centros educativos (Levine y Lesote, 1990; Creemers, 1994; Sammons et al., 1995; MacBeath y Mortimore, 2001; Murillo, 2005, 2006; Murphy et al., 2006; MacBeath y Dempster, 2008; Dimmock, 2012). Al mismo tiempo, los estudios sobre desarrollo y mejora escolar también han enfatizado la importancia de los líderes escolares para el mismo propósito (Leithwood, 1992; Bolam et al., 1993; Fullan, 1991, 1992, 1993; Hopkins et al., 1994, 1996; Reynolds et al., 1996; Huber, 1999; Leithwood, 2005; OCDE, 2008; Bush, 2009; Robinson et al., 2009). Sin dejar de tener en cuenta el peso del contexto sociocultural, esta perspectiva ha contribuido a subrayar el efecto decisivo de la dinámica de funcionamiento de las escuelas en sus resultados. Desde esta óptica, se contempla cómo los centros educativos, atendiendo a su forma específica de organización, pueden contribuir al desarrollo educativo de sus alumnos (Reynolds y Creemers, 1990). Aproximadamente entre un 12 y un $18 \%$ de la variación de los resultados de los alumnos pueden ser explicados por factores vinculados a la escuela y al aula (Creemers, 1994; Bell et al., 2003; Darling-Hammond y Rothman, 2011). La investigación desarrollada por algunos organismos internacionales sobre la mejora de la escuela (OCDE, 1982, 1986) también ha subrayado la capacidad organizativa del propio centro para incidir en el éxito educativo y ha interpretado la mejora escolar como un itinerario hacia el cambio en las condiciones de aprendizaje que debe permitir alcanzar los objetivos educativos de forma más eficaz (Miles y Ekholm, 1985; Hopkins y Ainscow, 1993; Bond, 2011).

Para seguir este itinerario, el liderazgo se ha contemplado como un factor crítico (OFSTED, 2000). La investigación ha mostrado su vinculación con la innovación y el éxito educativo (Van Zendel et al., 1985; Hopkins, 2001; West et al., 2000; McKinsey, 2010; Spillane y Coldren, 2011) en la medida que adquiere carácter instruccional y transformacional (Hopkins, 2001; Hallinger, 2003). Los directores capaces de liderar este proceso fijan sus principales objetivos en el ámbito pedagógico (Fullan, 2002; DarlingHammond et al., 2007; Townsend y MacBeath, 2011) y los persiguen a través de un proceso de reconstrucción cultural que llega a institucionalizarse para formar parte de la acción cotidiana. Esta orientación transformacional del liderazgo (Murphy, 1991;

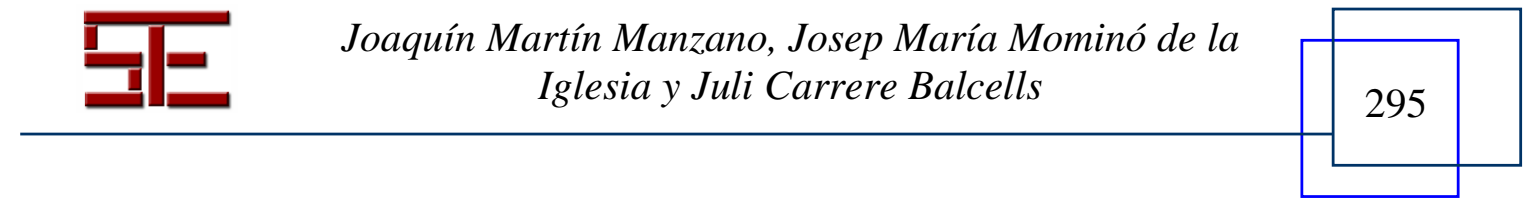




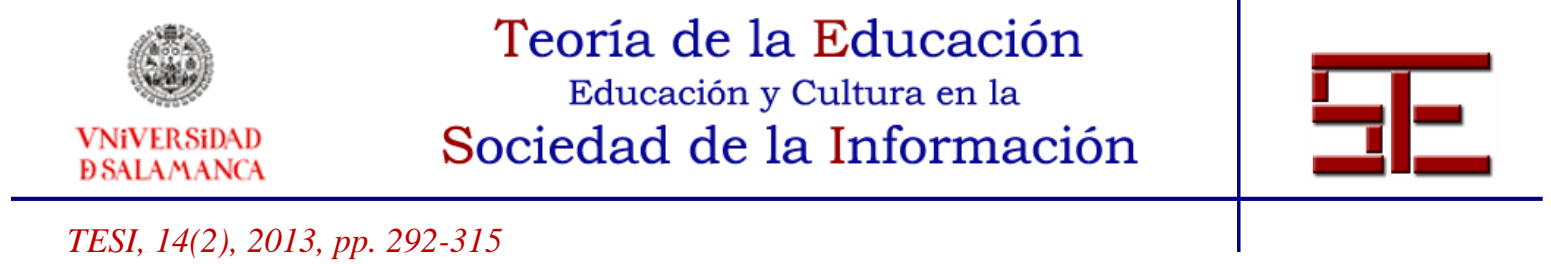

Leithwood, 1994; Leithwood, 2006) constituye un factor clave en la capacidad de afrontar el cambio y entender la mejora (Ainscow, Hopkins et al., 1994; Leithwood y Riehl, 2003; Leithwood, 2005; Hargreaves y Fink, 2006; Leithwood et al., 2006).

La orientación del cambio, de acuerdo con esta visión específica, debe materializarse en proyectos que permitan planificar la estrategia educativa a corto, medio y largo plazo (Bolívar, 2000; Antúnez, S., 2004; Gairín, J. y Muñoz, J.L., 2008). La administración de la estrategia, en términos de diseño del curso de acción, asignación de recursos e implementación, constituye el medio para concertar esfuerzos bajo un marco referencial consensuado que puede movilizar a los integrantes de la organización. En cualquier caso, la orientación de dicho diseño debe dirigirse a los cambios curriculares y a las prácticas docentes, tanto como a las estructuras organizativas y a los procesos necesarios para apoyar estos cambios (Robertson, Wohlstetter y Mohrman, 1994; Spillane, 2006; Robinson, 2007; Pont et al., 2008). La planificación estratégica, cuando se concreta en esta dirección, constituye un instrumento fundamental para un liderazgo centrado en el aprendizaje (Leithwood, 2007; Day, Sammons y Hopkins, 2009). Su análisis puede proporcionar indicios sobre el nivel de desarrollo alcanzado por esta forma de acción directiva en la vida cotidiana de los centros educativos.

\subsection{Presencia del liderazgo pedagógico en los centros educativos}

Una aproximación genérica a la implantación del liderazgo en el ámbito educativo seguramente pondría de relieve su tendencia marcadamente directiva, es decir, su carácter burocrático, técnico y autoritario, a pesar del interés que suscitan excepciones remarcables que escaparían a la tendencia general. El director, exceptuando estos casos, durante todo el período preconstitucional en España, centraba su función en la representación institucional ante algunos interlocutores sociales, el control del desarrollo de un currículum establecido y la disciplina del centro, así como en la gestión de la vertiente burocrático-administrativa de la escuela. Desde este punto de vista, la orientación pedagógica del liderazgo tuvo una presencia poco relevante en el planteamiento de la acción directiva establecida en ese período.

De hecho, la definición de la dirección de centros ha encontrado muchas dificultades para consolidar un modelo estable en el sistema educativo español. La definición técnicoburocrática de la dirección escolar se configura, en 1967, a través de las direcciones sin formación previa y la creación, el mismo año, del cuerpo de directores escolares. El reglamento de este cuerpo regulaba, en un contexto político y educativo autoritario y no

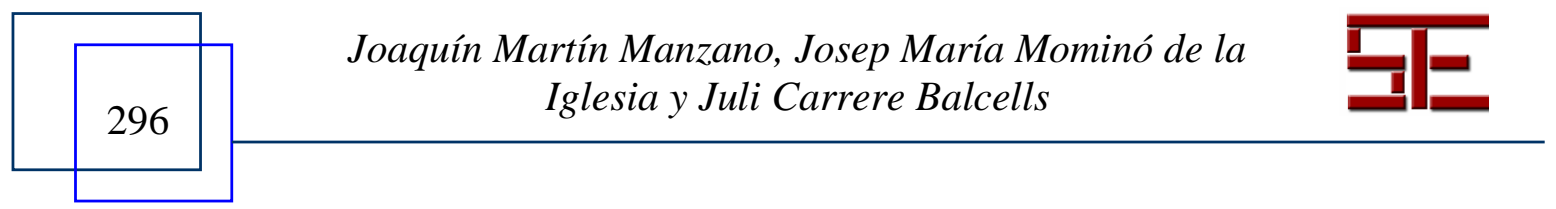




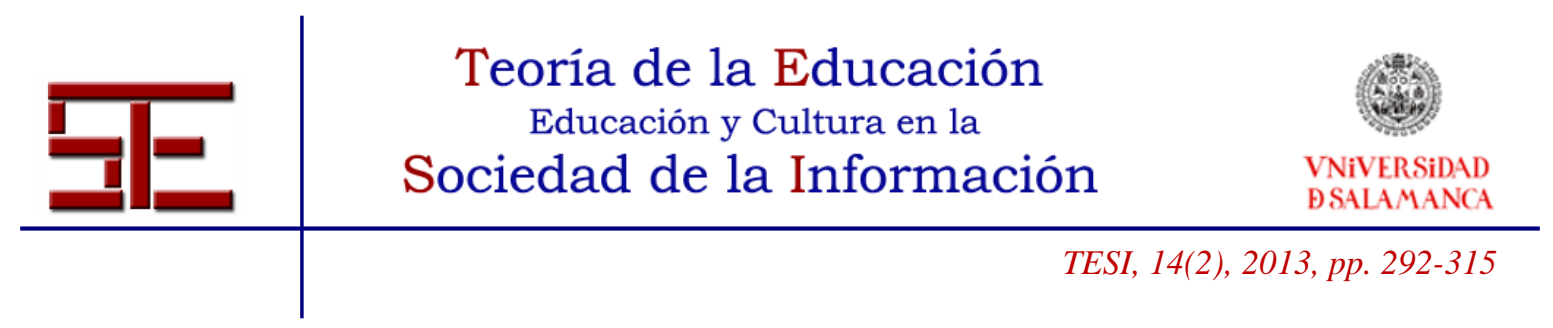

participativo, una dirección que conjuntaba estas mismas características con una clara profesionalización tecnocrática. Por suerte, fue un sistema que sólo duró tres años, hasta la Ley del 70 (LGE, 1970). El artículo 60 de esta ley establecía que el director sería nombrado por el Ministerio de Educación una vez escuchados el claustro y el consejo asesor. Ahora bien, no sería hasta 1981 que, con el Real Decreto 1.275/1.981, se estableció un verdadero sistema de propuesta por el claustro. La aprobación el 1985 de la LODE supuso la implantación en España de un modelo de dirección participativo, democrático y no profesional, escogido por el Consejo Escolar de centro, máximo órgano de gobierno colegiado de los centros docentes a partir de aquel momento. De esta forma se estableció el marco legal que posibilitó la configuración de un nuevo modelo para el ejercicio de la función directiva.

La alternancia en el ámbito político supuso en 1996 una nueva concepción de la función directiva, de tipo gerencial e influencia anglosajona, en que el director se concibió como un "manager" que gestionaba un centro teóricamente autónomo. El Documento de Bases de la "Ley de Calidad de la Educación" (2002) comportó la desaparición de la concepción participativa-democrática que suponía la elección del director por parte del consejo escolar del centro docente. Como alternativa, se propuso un modelo selectivo basado en méritos y la definición de un proyecto de dirección, sujeto a evaluación por parte de una comisión en que tienen representación padres, profesores y la propia administración educativa (un inspector y un director/a de otro centro). Este modelo ha sido ratificado y mejorado posteriormente por la LOE (2006) otorgando aún más fuerza al proyecto de dirección en consonancia con el proyecto educativo de centro y con las prioridades del sistema establecidas por la administración educativa.

Los estudios desarrollados por algunos organismos internacionales en la última década han contribuido a destacar el valor del liderazgo educativo. El Informe McKinsey (Barber y Mourshed, 2007) y la propia OCDE (Pont, Nusche y Moorman, 2008) consideraron que este es el segundo factor interno de la escuela que más contribuye a la mejora de los resultados de aprendizaje, siendo superado únicamente por la acción docente del profesorado. Asimismo, aproximaciones más recientes al liderazgo educativo (Day, Sammons y Hopkins, 2009; MacBeath y Nempster, 2009; Dimmock, 2012) continúan reclamando un modelo de profesional docente (learning-centered leadership) en que la orientación de la actividad directiva hacia la mejora del proceso de enseñanza y aprendizaje constituya un aspecto crítico. En consecuencia, aunque la cultura de la dirección escolar en España no se ha caracterizado, prioritariamente, por la orientación a la mejora de los resultados propia del liderazgo pedagógico o instruccional (TALIS,

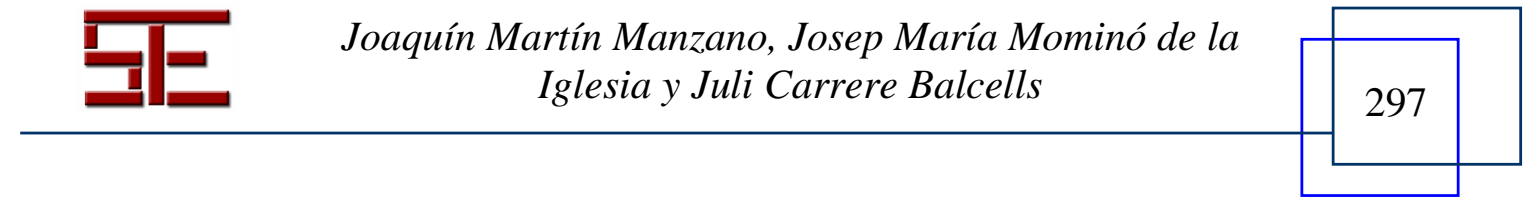




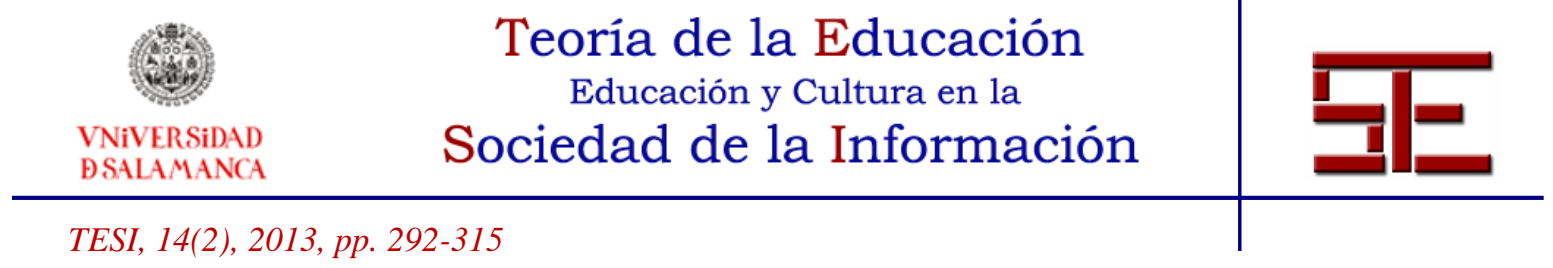

2009), la potencialidad de esta concepción va tomando cada vez más relieve, ante la insuficiencia de los planteamientos más centrados en la gestión técnica (Bolívar, 2010).

Este tránsito, desde un modelo burocrático al de dirección pedagógica, se ha ido reflejando progresivamente en la alternancia de las últimas regulaciones legislativas. La "Ley Orgánica de Educación" (LOE) subrayó (art. 132) que el director, en el ejercicio de sus funciones, debería "ejercer la dirección pedagógica, promover la innovación educativa e impulsar planes para la consecución de los objetivos del proyecto educativo del centro". Se subrayaba, de esta forma, el carácter estratégico de esta dirección pedagógica que debería poder traducirse en el diseño y aplicación de planes específicos que permitan el desarrollo de proyectos capaces de promover la mejora educativa. Esta forma de entender la dirección, asimismo, tiene su traducción específica, en la legislación desarrollada desde la administración educativa de algunas comunidades autónomas. Este sería el caso del Decreto de Autonomía de Centros de Catalunya (Decreto 102/2010, de 3 de agosto) que también incorpora el liderazgo pedagógico en su definición de la función directiva. En último término, el relieve que va tomando esta idea en las políticas educativas no debería extrañar si tenemos en cuenta el valor que se le concede para la mejora de la educación. En España, la potencialidad que se atribuye de forma progresiva a este liderazgo dirigido al aprendizaje posiblemente debe entenderse en un momento en que, a pesar de su desarrollo sostenido en las últimas décadas, los resultados educativos no han conseguido situarse de forma suficientemente satisfactoria en la comparativa con el conjunto de los países de la OCDE. Es desde este convencimiento que este artículo ofrece un análisis empírico sobre algunos de los indicios capaces de reflejar el nivel de desarrollo alcanzado por este tipo de liderazgo, en las etapas obligatorias de nuestro sistema educativo.

\section{2.- METODOLOGÍA DE INVESTIGACIÓN}

Los resultados que se presentan parten de los datos obtenidos en el proyecto de investigación La integración de internet en la educación escolar española: situación actual y perspectivas de futuro (Sigalés, C.; Mominó, J.M.; Meneses, J. y Badia, A, 2008), que analiza el proceso de integración de las tecnologías de la información y la comunicación (TIC) en los centros educativos de primaria y secundaria obligatoria (ESO) de España a través de las informaciones proporcionadas por los diferentes colectivos educativos implicados. Entre estos, la información facilitada por los directores de los centros nos permite profundizar en el estudio en la actividad de planificación estratégica que realizan en los centros.

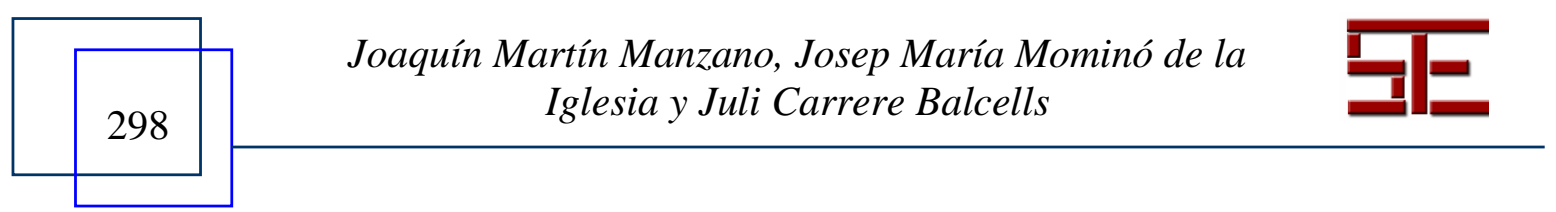




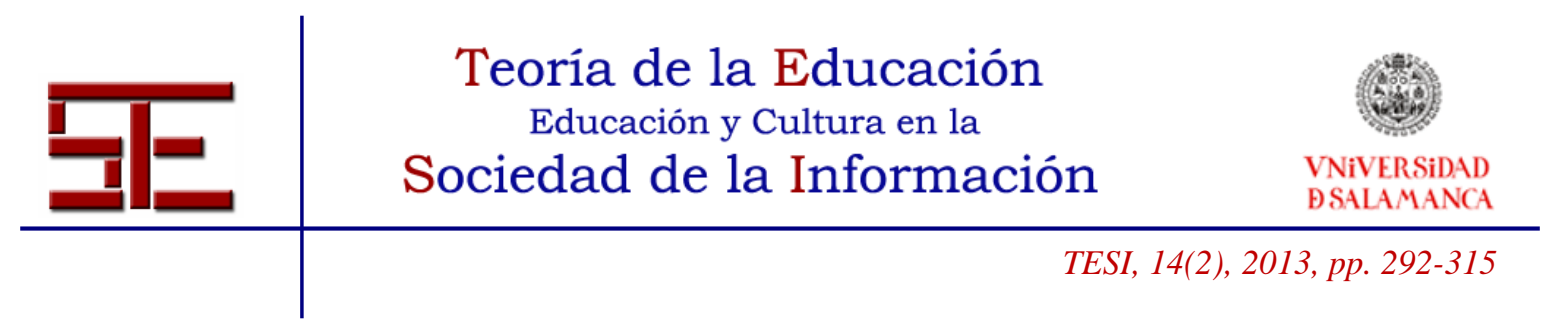

La investigación se ha desarrollado a través de una metodología cuantitativa en la que se ha utilizado el diseño de encuesta para recoger información a través de cuestionarios presenciales. El universo del estudio es el alumnado, el profesorado y los equipos directivos de los 17.986 centros que impartían estudios de educación primaria y secundaria obligatoria en España, durante el curso 2005-2006, según los datos facilitados por el Ministerio de Educación y Ciencia. Teniendo en cuenta este universo, se fijó en 800 el número de centros educativos para aceptar unos márgenes de error máximos para los resultados globales de $\pm 3,5 \%$, para un nivel de confianza del $95 \%$ y en el supuesto de máxima indeterminación $(\mathrm{p}=\mathrm{q}=0,50 \mathrm{y} \mathrm{k}=2)$. La segunda mitad del curso 2006-2007, se llevó a cabo un trabajo de campo que permitió entrevistar y recoger información de 809 centros que imparten educación primaria y secundaria obligatoria de España para completar una muestra de 1.697 profesores, 15.185 alumnos y 694 directores de centro. Estos últimos conforman la muestra del presente estudio.

La disponibilidad de esta base de datos con información detallada de los directores de centro ha permitido plantearse varios objetivos de estudio. En primer lugar, conocer la influencia del contexto institucional en la toma de decisiones materializada en los planes educativos. En segundo término, estudiar el efecto de las características de centro en el proceso de planificación. Finalmente, analizar la influencia de las características personales del director/a, tanto en aspectos socio demográficos, como en relación a las actitudes y creencias que favorecen un liderazgo pedagógico plasmado en la planificación pedagógica de los centros.

Como ya hemos indicado, el instrumento de recogida de la información ha sido un cuestionario dirigido específicamente a la persona que ejerce las funciones de dirección en el centro. El diseño del cuestionario se estructura en once apartados (disponible en http://www.uoc.edu/in3/integracion_internet_educacion_escolar/esp/informe.html). En el apartado denominado prioridades pedagógicas del centro, los directores de centro reportan el número de planes llevados a cabo los últimos tres años, entre un abanico de siete planes genéricos posibles. Esta fuente ha permitido construir el indicador de planificación estratégica. Así, aplicar tres o más planes se ha considerado un nivel moderado e incluso elevado de planificación; uno o dos planes una débil actividad de planificación, y la no aplicación de planes ofrece evidencias de la ausencia de esta forma de actividad estratégica en los aspectos específicos considerados en este indicador, aunque esto no excluya la posibilidad de planificación en otros ámbitos.

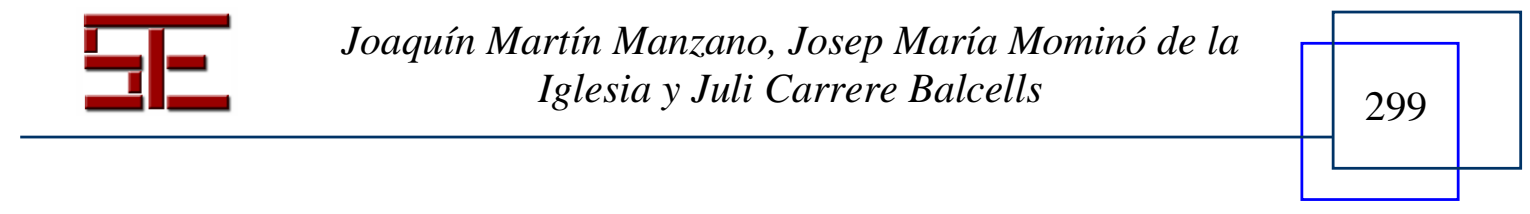




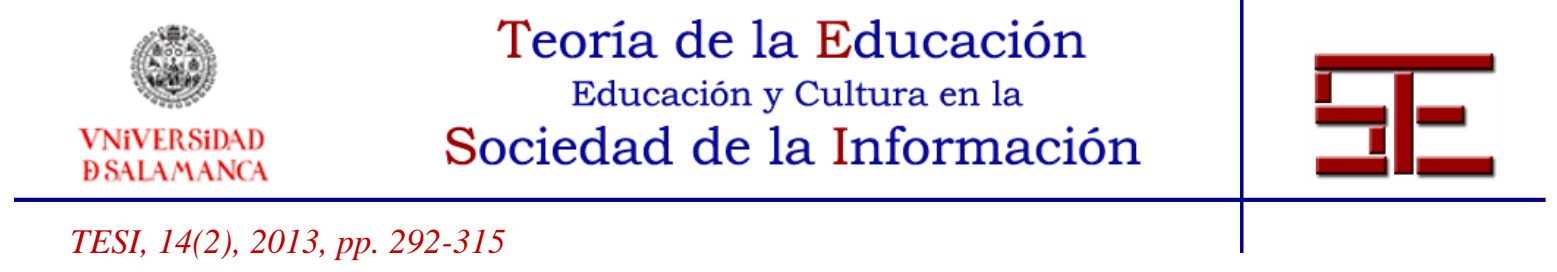

Este indicador ha sido analizado de modo descriptivo, tomando en cuenta la influencia de las características del contexto educativo: titularidad, tamaño del municipio, etapas educativas y confesionalidad. Por otro lado, se ha estudiado la asociación entre la planificación y las características del centro: número de alumnos, número de alumnos extracomunitarios y número de profesores. Finalmente, el indicador de planificación se ha relacionado, por un lado, con los datos socio-demográficos: edad, sexo, años de docencia y años de dirección. Por otro lado, se ha analizado la influencia de las creencias y las actitudes de los mismos directores en el proceso de planificación. Los datos obtenidos de los cuestionarios han sido tratados y analizados mediante el programa SPSS 19 para la obtención de los resultados estadísticos.

\section{3.- EL LIDERAZGO Y LA PLANIFICACIÓN ESTRATÉGICA}

El análisis toma el nivel de planificación estratégica como uno de los indicadores del liderazgo que se ejerce en los centros. La planificación puede ser considerada una actividad estratégica, en la medida que supone la definición de objetivos a alcanzar en plazos preestablecidos. En este sentido, el análisis intenta dilucidar la presencia de esta planificación, en algunas de sus principales vertientes. En último término, se trata de intentar calibrar cómo se mantiene el equilibrio entre el liderazgo administrativo y el liderazgo pedagógico. La situación en la que se encuentran los centros, en relación al nivel de planificación estratégica existente, en el ámbito pedagógico, puede ofrecer un indicador del nivel de desarrollo de las formas de liderazgo instructivo y transformacional, que consideramos críticos para la eficacia y la mejora de la actividad de los centros educativos.

Una primera aproximación a la actividad directiva de los centros y al indicador de referencia permite observar que, en una tercera parte de los casos $(31,6 \%)$, la planificación estratégica es nula o muy limitada: un 14,7\% de los directores no disponen de estos planes y un 16,9\% han desarrollado únicamente uno o dos. En cualquier caso, conviene poner la atención en la configuración de esta actividad estratégica, lo cual nos permitirá distinguir tres grupos, según su peso específico en el conjunto de la actividad que analizamos. Se trata, en todos los casos, de formas de planificación sobre aspectos pedagógicos que se pueden asociar con un tipo de liderazgo instruccional.

En el primer grupo, la actividad de planificación que se lleva a cabo con mayor frecuencia se refiere a acciones relacionadas con el acceso a la información de los alumnos y el apoyo externo al profesorado. Concretamente, casi tres cuartas partes de los directores (70\%) disponen de planes para fomentar habilidades de búsqueda de información en los

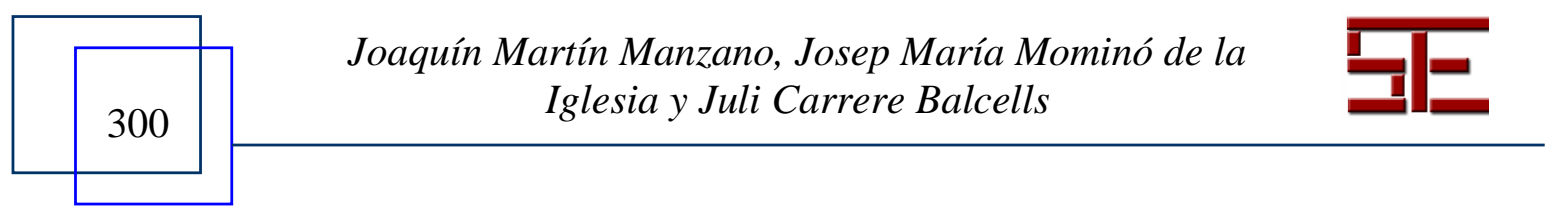




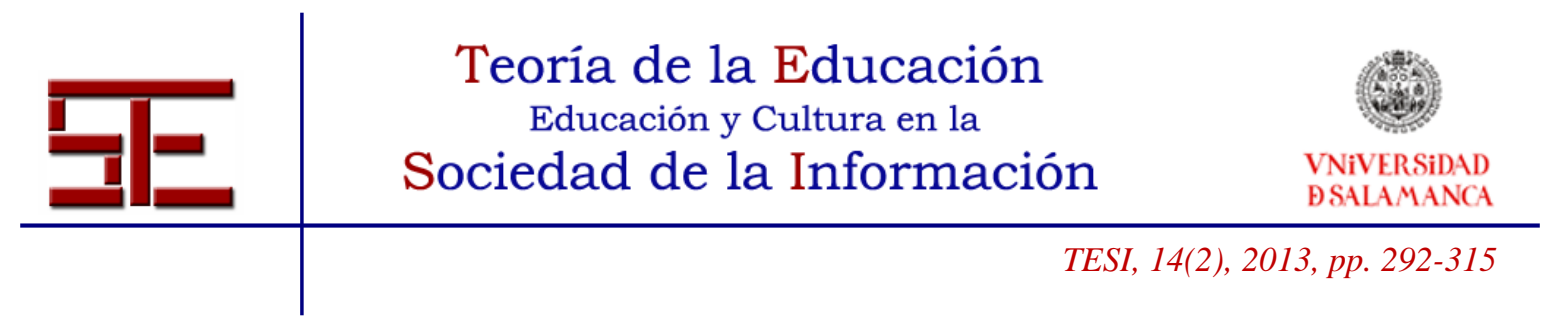

alumnos y también son mayoría $(62,8 \%)$, aunque en menor medida, los que utilizan alguna planificación para fomentar habilidades de aprendizaje independiente. Con una presencia similar $(60,3 \%)$, este grupo se completa con la planificación referida a la búsqueda de apoyo en agentes educativos externos para complementar el trabajo del profesorado.

Cuando la atención se dirige de forma más específica a la planificación estratégica para el espacio nuclear donde se desarrolla la actividad de enseñanza y aprendizaje, podemos apreciar que la frecuencia disminuye. Así, la mitad de los centros $(51,5 \%)$ disponen de planes para introducir una mayor personalización de la enseñanza. Un porcentaje parecido de escuelas $(50,5 \%)$ fomenta el desarrollo de proyectos interdisciplinarios que implican la colaboración entre profesores durante un periodo largo de tiempo. Así, pues, parece interesante el número de centros que ya están comprometidos en este tipo de actividad para la mejora de la práctica pedagógica en el aula; sin embargo, hay que destacar también la mitad complementaria en que no se ha podido detectar esta estrategia que puede considerarse crítica para un liderazgo orientado al aprendizaje.

Finalmente, cuando la atención se dirige a aquellas formas de planificación que pretenden poner al alumno en el centro del proceso de aprendizaje (OECD, 2008, 2010) se constata inmediatamente que su presencia es aún menor. Los planes para incrementar la participación de los alumnos en las decisiones sobre su propio proceso de aprendizaje (sobre temas a estudiar, ritmo de trabajo, tipo de actividades...) ya no se pueden encontrar en la mayoría de los centros $(41,2 \%)$. En último término, no llegan a ser una tercera parte (31\%) los directores que impulsan planes para fomentar el desarrollo de proyectos de larga duración que implican la colaboración entre alumnos, durante un periodo largo de tiempo. En este sentido, se puede constatar que las formas de planificación que más se podrían vincular a un liderazgo pedagógico, centrado en facilitar las condiciones que pueden favorecer el protagonismo de los alumnos en su proceso de aprendizaje, son las que tienen un protagonismo menor.

\subsection{La planificación estratégica según el contexto y las características del centro}

Ahora bien, a partir de aquí conviene profundizar en los datos disponibles con el propósito de intentar asociar variables contextuales con el tipo de liderazgo ejercido. Para este propósito, el análisis recurre a dos aspectos específicos que pueden informar sobre la incidencia del entorno en la dinámica de funcionamiento del centro: el tamaño del

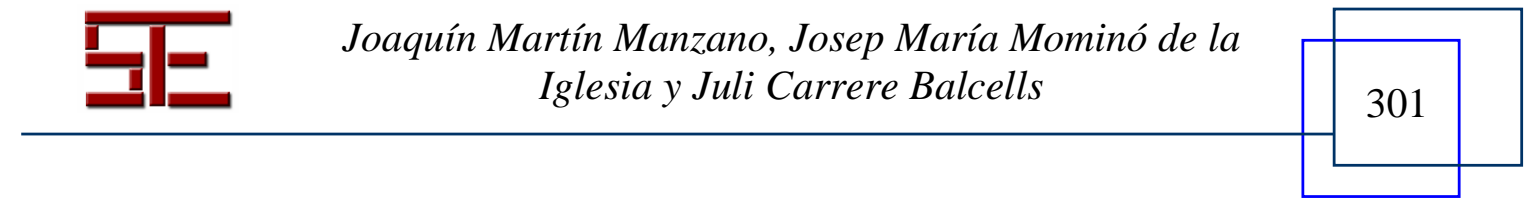




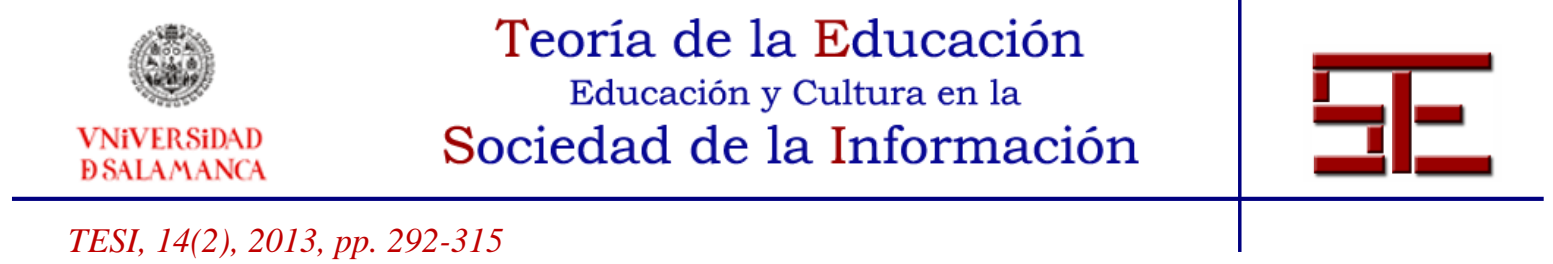

municipio en que se ubica y la presencia de alumnado extracomunitario. Así, inicialmente, no se puede apreciar ninguna vinculación entre la actividad de planificación estratégica y estos factores referidos al contexto: no se identifican diferencias significativas entre centros ubicados en municipios pequeños, medianos o aquellos que pertenecen a las ciudades con mayor número de habitantes. Al mismo tiempo, el número de alumnos extracomunitarios del centro tampoco permite apreciar ninguna tendencia significativa. La composición sociocultural, en este sentido, no parece tener una clara incidencia en el desarrollo de la actividad estratégica que se analiza.

Más allá de estos condicionantes de entorno, una mayor aproximación a la singularidad de los centros puede mostrar en qué medida su titularidad, pública o privada, se traduce en fórmulas específicas de organización y gestión. En este aspecto, se constata que más de dos terceras partes $(65 \%)$ de los centros públicos presentan una actividad de planificación moderada e incluso elevada. No obstante, no es despreciable la tercera parte restante en que el nivel de planificación es bajo $(18,2 \%)$ o nulo $(16,8 \%)$ en los aspectos críticos que analiza este indicador. En cambio, el contraste con los centros privados permite observar que esta fracción menos activa se reduce significativamente $(8,6 \%)$ y acaba siendo minoritaria. Así pues, en los centros de titularidad privada, más de tres cuartas partes $(78,2 \%)$ desarrollan esta forma de actividad estratégica. Se constata así que la titularidad se asocia a diferencias estadísticamente significativas en cuanto al nivel de actividad de planificación a que nos estamos refiriendo.

Cuando se observa la relación con la confesionalidad de los centros, los resultados muestran una tendencia similar y esperable, dado el carácter laico de los centros públicos. En efecto, la confesionalidad del centro también permite establecer diferencias significativas. Los centros religiosos muestran una disposición a la planificación estratégica más intensa que la de los centros laicos. Entre estos, una gran mayoría $(76,1 \%)$ de-sarrollan planes con frecuencia. En cambio, esta actividad estratégica referida a los aspectos pedagógicos del centro se reduce a dos terceras partes en los centros laicos $(66,3 \%)$.

Trasladando la atención al tamaño y estructura organizativa del centro, las diferencias entre centros siguen siendo manifiestas. Observando las etapas educativas impartidas, el análisis puede mostrar en qué medida la complejidad de la estructura organizativa se puede relacionar con el tipo de planificación o la intensidad con que se ejerce. En los centros de estructura organizativa más compleja, que ofrecen primaria y secundaria, los directores se muestran más activos $(78,7 \%)$ en acciones de planificación. Este porcentaje

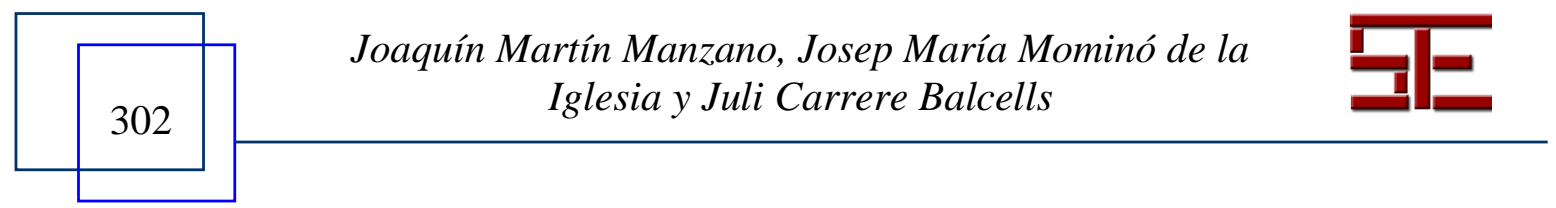




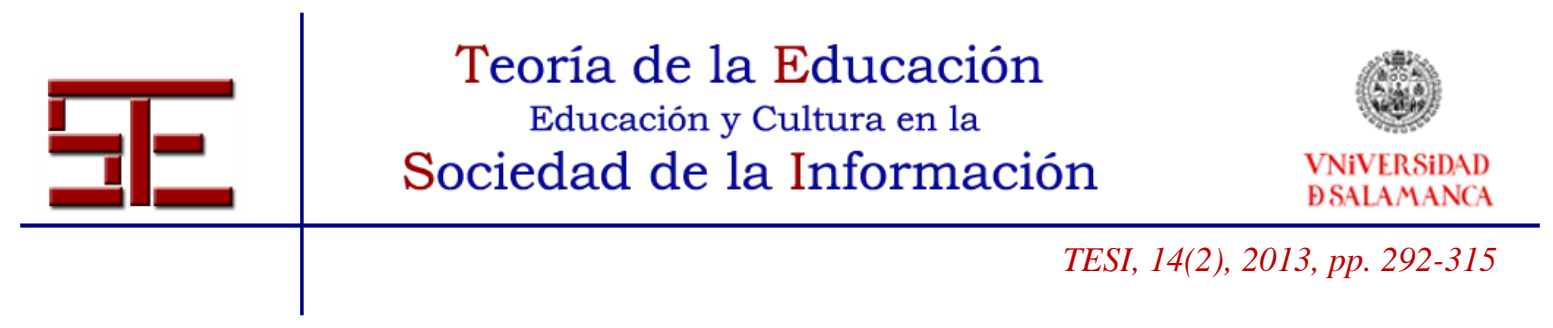

se reduce en los centros que solo ofrecen educación secundaria $(71,4 \%)$ y más aún en los centros de primaria $(62,6 \%)$. Así, estos últimos son los menos activos en términos de planificación: un 18,6\% de estas escuelas no impulsan ninguno de los planes a que se refiere el análisis. En cambio, este porcentaje se reduce a la mitad $(9,5 \%)$ en los centros que ofrecen ambas etapas educativas. Estos resultados son nuevamente coincidentes con los observados en términos de titularidad, puesto que los centros que imparten todas las etapas de la educación obligatoria son de titularidad privada en nuestro sistema educativo.

Los resultados observados en relación al número de alumnos y profesores abundan en la misma dirección. Quizás sea remarcable que un $62,7 \%$ de los centros más pequeños han desarrollado 3 o más planes y que este porcentaje se va incrementando ligeramente en los centros con más alumnado, hasta aproximadamente las tres cuartas partes (73,2\%). Estos resultados son coincidentes con el número de profesorado del centro, lo cual es lógico, dado que ambas variables tienen que ver con el tamaño del centro. Aproximadamente, tres cuartas partes $(70,9 \%)$ de los centros más grandes intensifican su planificación bajo la perspectiva analizada. En definitiva, en los centros de mayores dimensiones la planificación estratégica tiene una presencia significativa mayor y el tamaño del centro se asocia con la necesidad de planificación.

\subsection{La planificación estratégica según las características personales y las creencias de los directores}

A pesar del énfasis que se ha dado tradicionalmente a la asociación entre las características personales del director y su capacidad de liderazgo, desde ópticas basadas en los rasgos del líder o "teorías del gran hombre" (Stogdill, 1948, 1950; Burns, 1978; Yukl, 1981; Aguirre y Villarreal. 2012), el análisis no permite identificar diferencias asociadas a estos rasgos personales. Ni la edad, ni el género, ni la experiencia en docencia y dirección han podido asociarse a la actividad de planificación que estamos observando. Así, pues, sin menospreciar el valor que puede tener el carisma personal de los directivos en esta dinámica, no parece que el peso de este factor acabe siendo decisivo.

No obstante, más allá de estas características personales, la concepción de estos directores sobre cuestiones como el apoyo que encuentran en la comunidad educativa y las prioridades que identifican para el desarrollo de su responsabilidad cotidiana, parecen ser aspectos que, en este caso, sí se vinculan al nivel de desarrollo a que puede llegar la actividad estratégica de planificación. La representación que ofrecen los directores sobre

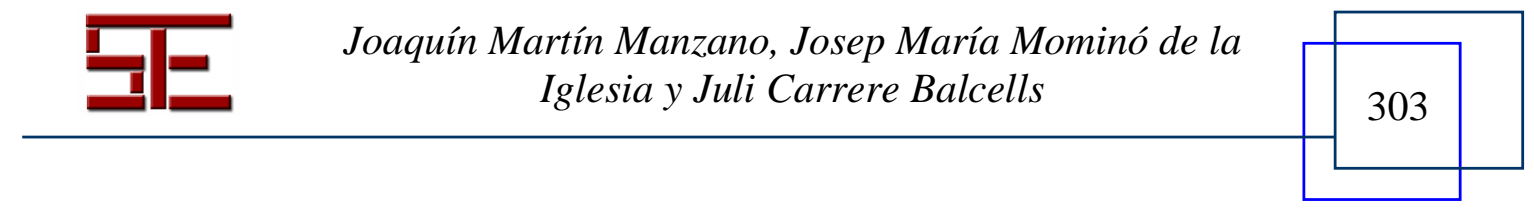




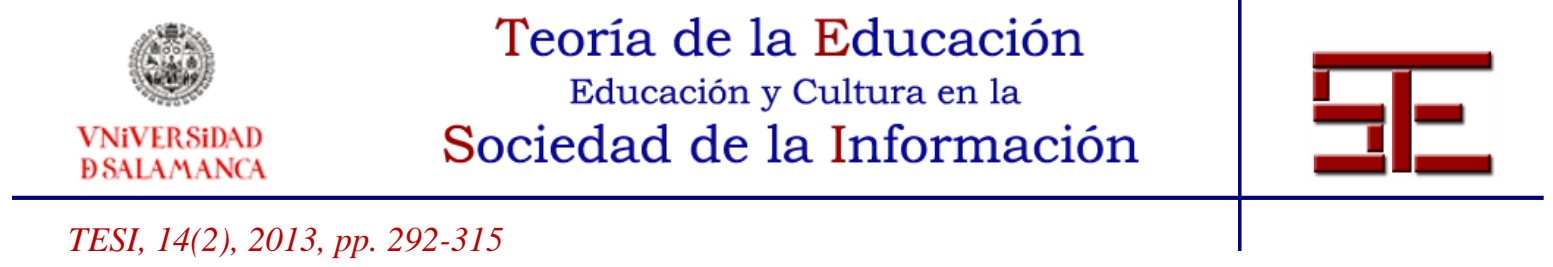

las circunstancias en que se desarrolla su actividad en el centro y específicamente el grado en que se orientan a la mejora de los procesos de enseñanza y aprendizaje encuentra su traslación en términos de iniciativa para un liderazgo pedagógico. El análisis del peso específico de estas creencias, relacionadas con una forma de liderazgo centrada en el aprendizaje y la mejora de los resultados académicos, permite observar la asociación a que nos estamos refiriendo.

En primer término, se pone de relieve el efecto de la percepción de los directores en relación al apoyo y grado de confianza que encuentran en su entorno inmediato y también a la potencialidad que atribuyen a su equipo de colaboradores próximo. En este sentido, inicialmente se observa que aquellos que se sienten más apoyados en instancias superiores son también los más activos (70,3\%). En cambio, cuando esta percepción de apoyo disminuye, la participación en los niveles elevados de planificación también se ve ostensiblemente mermada $(52,4 \%)$. En este mismo sentido, se distingue una relación significativa con el apoyo que encuentran en la comunidad escolar del propio centro. Casi la mitad de los directores $(45,5 \%)$ que no sienten este apoyo son también los que no han desarrollado ningún tipo de plan de acción. En contraste, cuando la sensación de apoyo de la comunidad es mayor, también aumenta el nivel de iniciativa. Más de dos terceras partes de los directores $(69,7 \%)$ que cuentan con este nivel elevado de confianza se encuentran entre los más activos en este proceso.

El nivel de seguridad a que nos estamos refiriendo también se puede observar en relación a la predisposición de los directores a asumir riesgos en la promoción de la innovación y el cambio en el centro, contemplando incluso el error como posibilidad. Nuevamente, la gran mayoría de los directores que cuentan con esta actitud $(70,7 \%)$ son también los que han elaborado un mayor número de proyectos. En cambio, los indiferentes $(53,4 \%)$ y los menos proclives a asumir los desafíos que supone la innovación son, a su vez, los menos proactivos $(50 \%)$. Asimismo, se constata que la confianza en las posibilidades de colaboración con un equipo directivo que ejerce un liderazgo sólido e influyente en el centro también favorece este nivel de iniciativa. Entre los que han articulado un equipo que supone un apoyo efectivo para la estrategia directiva, nuevamente la gran mayoría $(69,6 \%)$ se encuentran en los niveles de planificación más elevados. En cambio, los que no disponen de esta percepción de apoyo en su equipo directivo se encuentran en menor medida $(36,4 \%)$ entre los directores más activos.

Finalmente, la atención se dirige de forma específica al liderazgo pedagógico, es decir, al que encuentra su principal manifestación en el impulso de los procesos de aprendizaje de

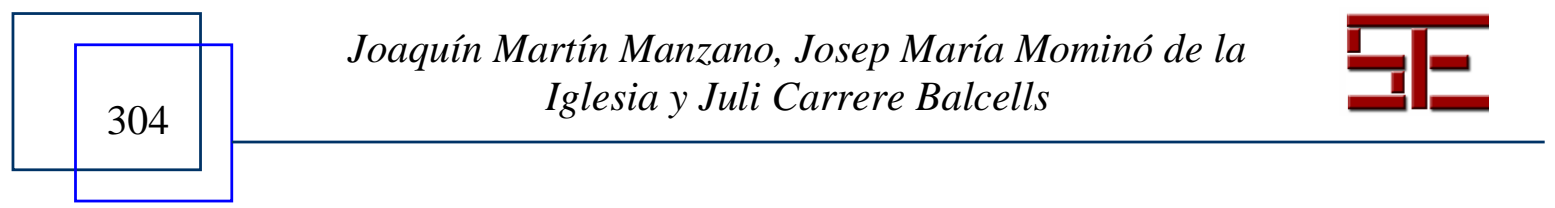




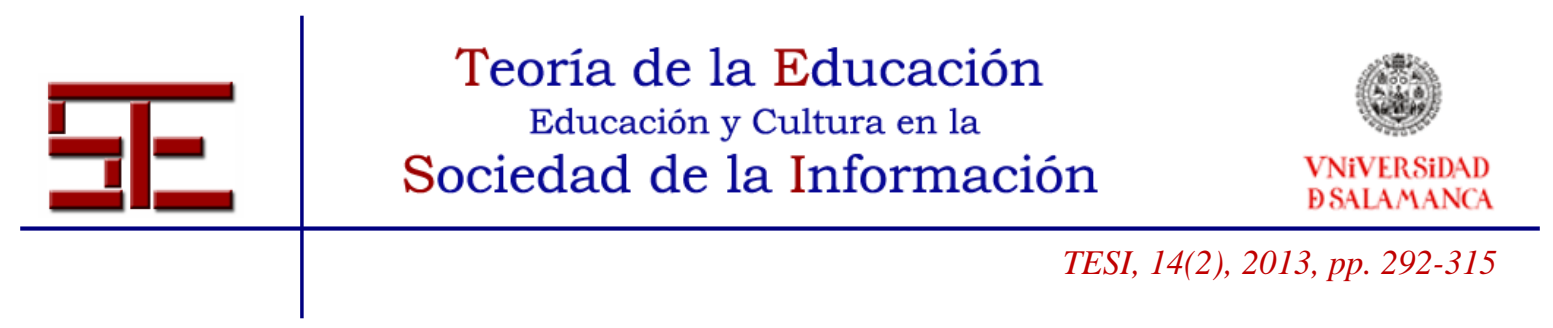

los alumnos para la mejora, en última instancia, de su rendimiento académico. En este aspecto, se constata que casi tres cuartas partes $(73,9 \%)$ de los directores para los cuales influir en el trabajo que lleva a cabo el profesorado en sus aulas es una función prioritaria se encuentran entre los más activos en el desarrollo de proyectos para el impulso de la actividad pedagógica. Los que conceden menor prioridad a esta función tienen una presencia menor $(60,8 \%)$ entre los directores con mayor iniciativa. En coherencia con estos resultados, también se puede observar como los directores que consideran que su trabajo repercute de forma significativa en la calidad de los aprendizajes que realizan los alumnos y en los resultados que obtienen se encuentran también, de forma muy mayoritaria $(73,8 \%)$, entre los más dinámicos. En cambio, entre los directores para los cuales la atención al aprendizaje no se sitúa en el primer plano de la acción directiva, el porcentaje de los más activos $(47,6 \%)$ disminuye claramente.

\section{4.- DISCUSIÓN Y CONCLUSIONES}

La capacidad de actuación de los centros educativos, a través de fórmulas de liderazgo pedagógico o instruccional, es decir, de aquellas formas de acción directiva que se dirigen específica y prioritariamente a los procesos de aprendizaje, se reconoce como uno de los principales mecanismos de influencia a través de los cuales las escuelas pueden llegar a contribuir a la mejora de los resultados académicos de sus alumnos. Las características de la planificación estratégica que se promueve desde la dirección de los centros educativos pueden ofrecer evidencias sobre el nivel alcanzado por este tipo de liderazgo y sobre algunos de los factores asociados a su desarrollo. La confianza en la eficacia de esta forma de acción directiva justifica el interés de una aproximación empírica a esa tarea estratégica en la actividad cotidiana de las escuelas.

El análisis pone de manifiesto que la planificación estratégica a que nos referimos no constituye una práctica extendida en una amplia mayoría de los centros educativos. En aproximadamente la mitad de las escuelas no se puede detectar esta orientación estratégica hacia el núcleo de la práctica pedagógica. De hecho, precisamente la actividad que se desarrolla en el aula y la participación del alumnado son los aspectos a que conceden menor atención estas formas de planificación. En este sentido, el liderazgo pedagógico puede ser considerado incipiente. Su presencia parece muy limitada en una amplia fracción de escuelas, donde el objetivo estratégico principal, en la práctica, no se traduce en la planificación de la mejora del proceso educativo. Esta orientación específica del liderazgo no parece totalmente independiente de las características del centro. El análisis permite constatar que la titularidad de los centros puede asociarse con la

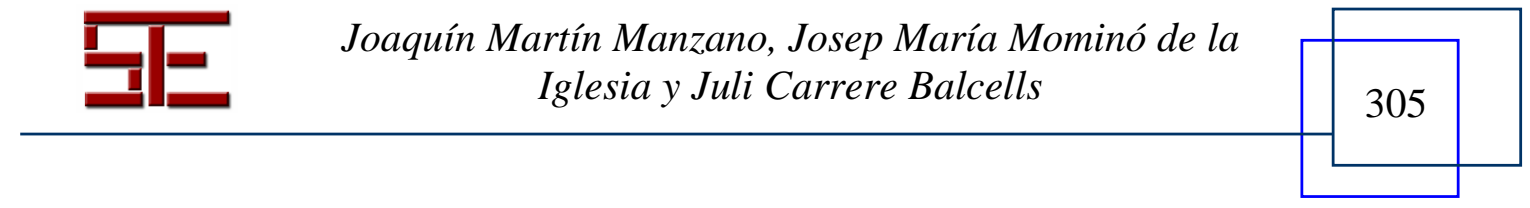




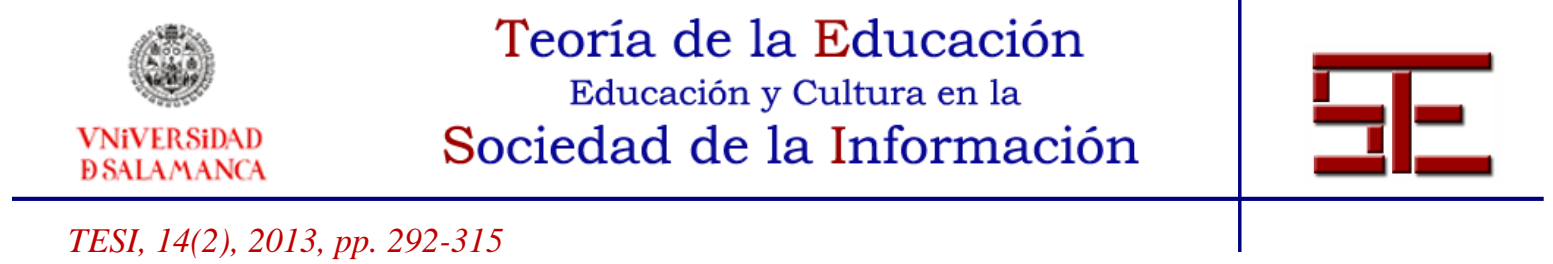

configuración de este proceso. La comparación entre centros privados o públicos, teniendo en cuenta distintos aspectos asociados a la titularidad, permite constatar, en estos últimos, un menor desarrollo de este tipo de acciones, propias de una dirección focalizada en la mejora de la práctica pedagógica.

Sin embargo, el análisis también permite subrayar que, en la cuestión que estamos analizando, la titularidad no debería confundirse con las características personales de los directores que no han podido asociarse con la forma de dirección a que nos estamos refiriendo. Aunque, seguramente, no se debe menospreciar la contribución singular de la persona responsable de la dirección, sus características personales no reflejan una vinculación significativa con el tipo de liderazgo ejercido. La forma de liderazgo a que nos estamos refiriendo, en cambio, muestra mayor relación con las características del propio centro educativo, con la situación específica y las creencias con que la propia dirección lleva a cabo su responsabilidad cotidianamente. En este sentido, la confianza percibida en el entorno inmediato y la posibilidad de apoyo en el equipo de colaboradores próximo, parecen ofrecer un escenario más propicio para un liderazgo pedagógico y, desde este punto de vista, también distribuido. En último término, el análisis pone de manifiesto la asociación entre las creencias de los directores y la inclinación de la acción directiva como mecanismo de influencia en la práctica pedagógica.

Los resultados de esta investigación ponen de manifiesto una situación de déficit y la necesidad de potenciar las formas de dirección que más pueden contribuir a la mejora del rendimiento académico de los centros educativos. La administración educativa, para este propósito, afronta el reto de impulsar la extensión en el sistema educativo de un liderazgo orientado prioritariamente al aprendizaje de los alumnos, especialmente en los centros de titularidad pública, donde como hemos visto el desafío es más importante. Parece que el desarrollo reciente de las políticas educativas es cada vez más sensible a la necesidad de contribuir a este proceso que, según muestran las evaluaciones internacionales (TALIS, 2009), en nuestro sistema educativo se encuentra en las posiciones menos avanzadas. Se trata de favorecer el modelo directivo desde donde se puede promover esta orientación de liderazgo en educación, estableciendo las condiciones para su desarrollo que el informe McKinsey (Barber y Mourshed, 2007) definía en términos de estructuración de funciones, definición de incentivos y expectativas.

Así pues, la planificación estratégica ofrece un indicador para la detección de la forma de liderazgo educativo a que se concede mayor potencialidad por su vinculación con la mejora del rendimiento académico. En cualquier caso, el papel clave de esta orientación

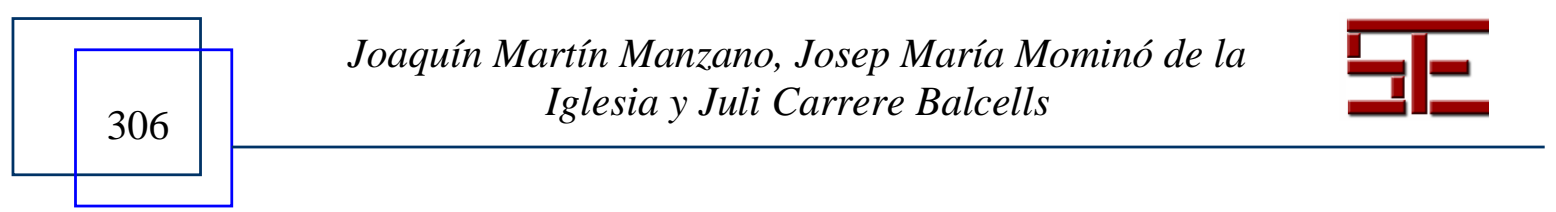




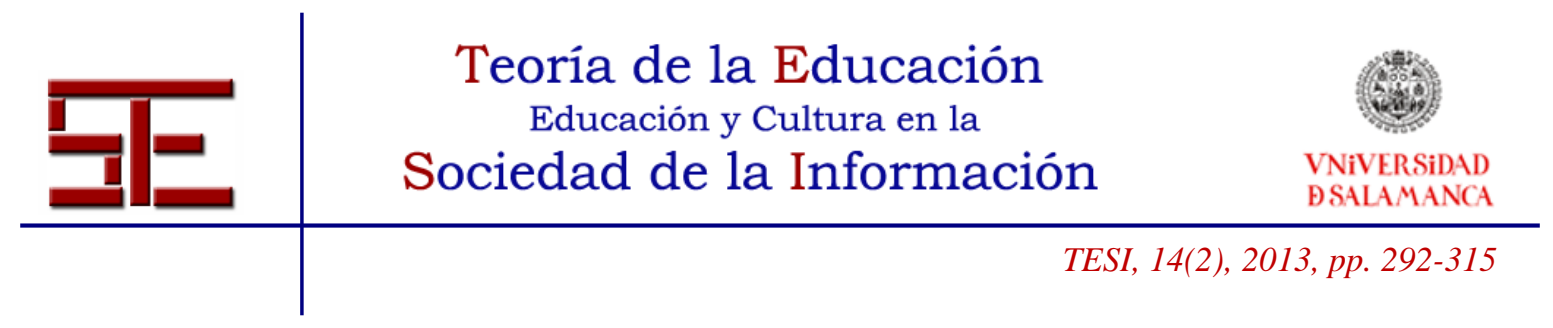

de la dirección escolar para alcanzar el éxito educativo de los alumnos y la eficiencia organizativa, así como el nivel de desarrollo incipiente que ha alcanzado en el sistema educativo, permiten anticipar que el análisis de este proceso se mantendrá como reto para la investigación futura. Ofrecer evidencias empíricas y representativas sobre los factores asociados a un liderazgo centrado en el aprendizaje y sobre su vinculación con los resultados educativos continuará siendo necesario no sólo para el diagnóstico, también para contribuir a identificar los componentes de la fórmula de dirección capaz de promover proyectos educativos orientados al éxito educativo de todos los alumnos.

En nuestro contexto inmediato, tropezamos aún con demasiada frecuencia con formas de dirección técnica propias de un modelo centralizado y burocratizado que responde prioritariamente a demandas administrativas externas. La subordinación de la acción directiva al aprendizaje del alumnado y, en consecuencia, a la mejora de la intervención educativa del profesorado constituye todavía un desafío para el sistema educativo. La profesionalización de la función directiva y la configuración de un estatus profesional adecuado para la dirección de centros serán condiciones decisivas para facilitar este proceso. El desarrollo reciente de la legislación en educación debería contribuir a crear estas condiciones. Actualmente, el anteproyecto de ley que propone la LOMCE (art. 122 y art. 132) contempla nuevamente, de forma genérica, la potenciación de la función directiva y de la dirección pedagógica. Aunque no parece resolverse con una fórmula definitiva la situación del estatus profesional y de profesionalización del director. No obstante, en último término, acaban siendo las formas específicas de actuación de la dirección, tal y como se concretan cotidianamente en cada centro, en base a la situación inmediata y a concepciones específicas sobre la función directiva, las que se traducen en acciones y formas distintas de liderazgo con una potencialidad igualmente distinta para la mejora de la práctica pedagógica. Es en este sentido que la investigación sobre las acciones de planificación estratégica desarrolladas por una muestra representativa de directores de nuestro sistema educativo puede ofrecer un indicador relevante del nivel de desarrollo a que ha llegado el liderazgo pedagógico en nuestro contexto inmediato, pero también de los retos a alcanzar para la dirección de la escuela del futuro.

\section{5.- BIBLIOGRAFÍA}

Altricher et al., (1998). Images of Educational Change, (99-110). Buckingham: Open University Press.

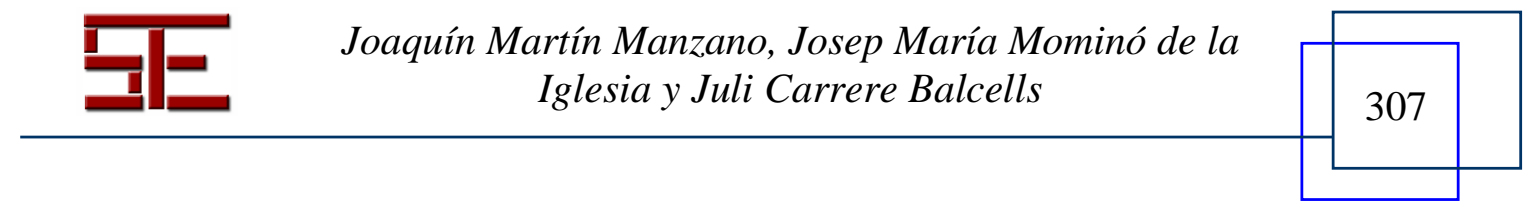




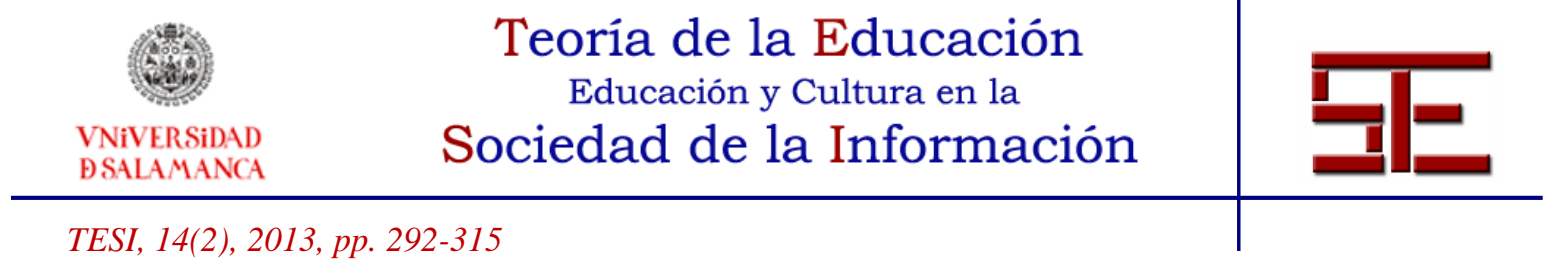

Álvarez, M. (2004). El liderazgo facilitador del cambio a través del itinerario formativo. En A. Villa (Coord.), Dirección para la Innovación: Apertura de los centros a la sociedad del conocimiento. Bilbao: Universidad de Deusto.

Antúnez, S. (2004). Organización escolar y acción directiva. México: SEP.

Barber, M. \& Mourshed, M. (2007). Informe Mckinsey.

Bell, L., Bolam, R. and Cubillo, L. (2003). A systematic review of the impact of school leadership and management on student outcomes. London: Research Evidence Library.

Bolam, R., McMahon, A., Poklington, K. \& Weindling, D. (1993). Effective Management in Schools. London: HMSO.

Bolívar, A. (1997). Liderazgo, mejora y centros educativos. En A. Medina. (Coord.): El liderazgo en educación, (pp. 25-46). Madrid: UNED.

Bond, N. (2011). Preparing preservice teachers to become teacher leaders. Educational Forum, 75, 280-297.

Brookover et al. (1979). School social systems and student achievemennt: schools can make a difference. New York: Praeger.

Bush, T. (2009). Leadership development and school improvement. Educational Review. London: Routledge.

Caldwell, B. J. \& Spinks, J. M. (1992). Leading the self-managing school. London: The Falmer Press.

Creemers, B. P. M. (1994). The History, Value and Purpose of School Effectiveness Studies. En D. Reynolds. et al., (Eds.), Advances in School Effectiveness Research and Practice. Oxford: Pergamon.

Creemers, B. L. P (1994). The Effective Classroom. London: Cassell.

Dalin, P. y Rolff, H. G. (1990). Das Institutionelle Schulentwicklungsprogramm. Soester Verlag-Kontor: Soest.

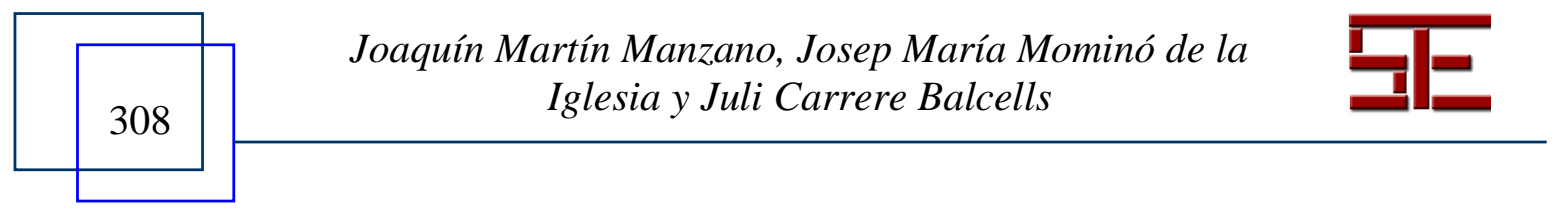




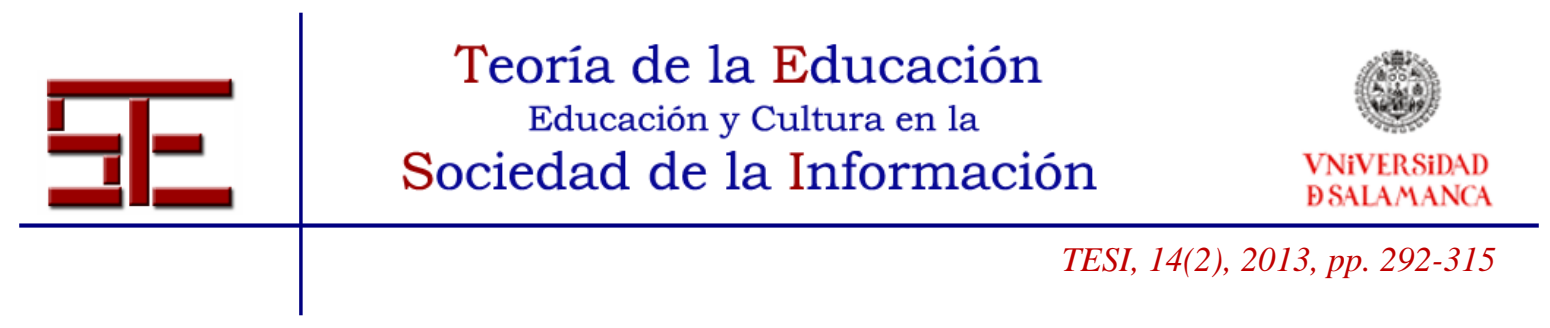

Darling-Hammond, L., LaPointe, M., Meyerson, D., \& Cohen, C. (2007). Preparing school leaders - lessons from exemplary leadership development programs. Wallace Foundation Report, accessed from http://seli.stanford.edu.

Darling-Hammond, L. and Rothman, R. (2011). Teacher and Leader Effectiveness in High-Performing Education Systems. USA, Washington, D.C.: Alliance for Excellent Education y Stanford, CA: Stanford Centre for Opportunity Policy in Education.

Day, C., Sammons, P., Hopkins, D., Harris, A., Leithwood, K., Gu, Q., Penlington, C.,

Mehta, P. y Kington, A. (2009). The impact of school leadership on pupil outcomes. Nottingham: National College for School Leadership.

Decret Autonomia. DECRET 102/2010, de 3 d'agost, d'autonomia dels centres educatius.

Decret de Direccions. DECRET 155/2010, de 2 de novembre, de la direcció dels centres educatius.

Dimmock, C. (2012). Leadership, capacity building and school improvement. London: Rouledge.

Edmonds, R. (1979). Effective schools for the urban poor. Educational Leadersbip, 37, $15-24$.

Fullan, M (1991). The new meaning of educational change. London: Cassell.

- (1992). Successful School Improvemen. Buckingham: Open University Press.

- (1992). Visions that blind. Educational Leadership, 49, 19-20.

- (1993). Change Forces: Probing the Depths of Educational Reform. London:

Falmer Press.

- (2002). The change leader. Educational Leadership, 59, 16-20.

Gairín, J. \& Muñoz, J. L. (2008). El agente de cambio en el desarrollo de las organizaciones. Enseñanza, 26, 187-206.

Hallinger, P. (2003). Leading educational change: Reflections on the practice of instructional and transformational leadership. Cambridge Journal of Education. Cambridge: Taylor \& Francis.

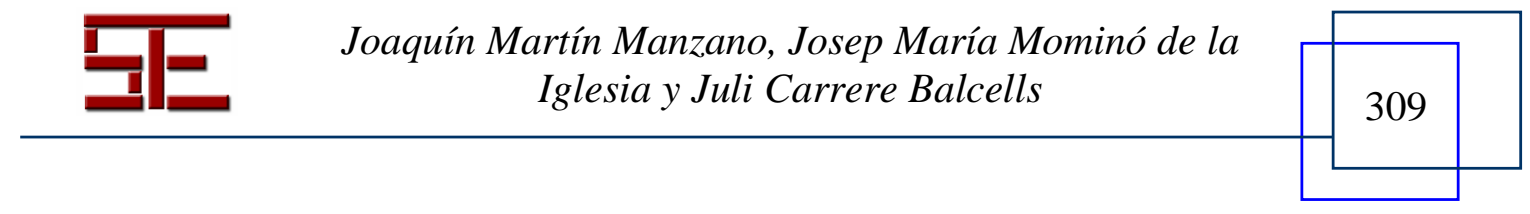




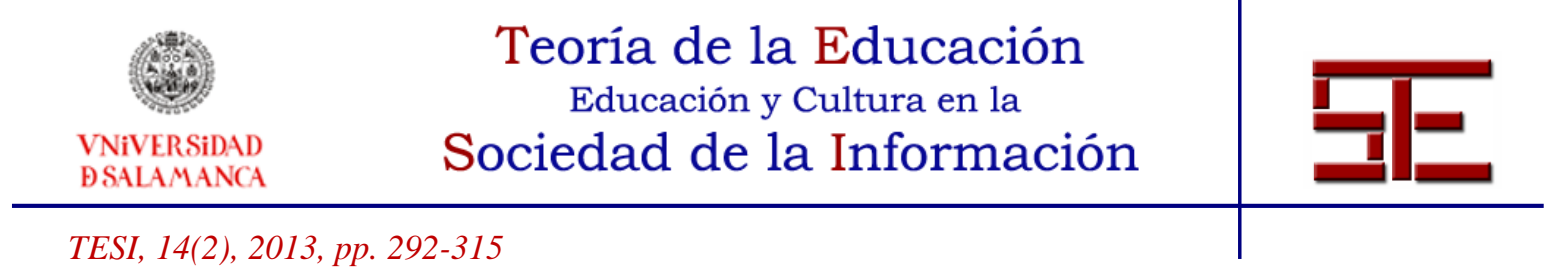

Hargreaves, A. \& Fink, D. (2006). Sustainable Leadership. San Francisco: Jossey-Bass.

Hopkins, D. (2001). School Improvement for Real. London: Falmer Press.

Hopkins, D. \& Ainskow, M. (1993). Making Sense of School Improvement: An Interim Account of the "Improving the Quality of Education for All" Proyect, Cambridge Journal of Education, 23 (1), 287-304.

Hopkins, D., Ainskow, M., \& West, M. (1994). School Improvement in an Era of Change. London: Cassell.

Hopkins, D., Harris, A. y Jackson, D. (1997). Understanding the School's Capacity for Development: Growth States and Strategies. School Leadership and Management, vol. $17,3,401-411$.

Huber, D. (1999). Liderazgo y administración en enfermería. México D.F.: McGraw-Hill Interamérica.

Huberman, M. (1992). Teacher development and instructional mastery. En A. Hargreaves \& M. G. Fullan. (Eds.), Understand Teacher Development (pp. 122 -142). New York: Teachers College Press.

Joyce, B. (1991). The doors to school improvement. Educational Leadership, vol. 48, 8, 59-62.

LEC. LEY 12/2009, de 10 de julio, de educación.

Leithwood, K. (1992). The move toward transformational leadership. Educational Leadership, 49, 8-13.

- (1994). Leadership for school restructuring. Educational Administration Quarterly, 30, 498-518.

- $\quad$ (1994). Liderazgo para la reestructuración de las escuelas, Revista de Educación, 304 (mayo-agosto), 31-60.

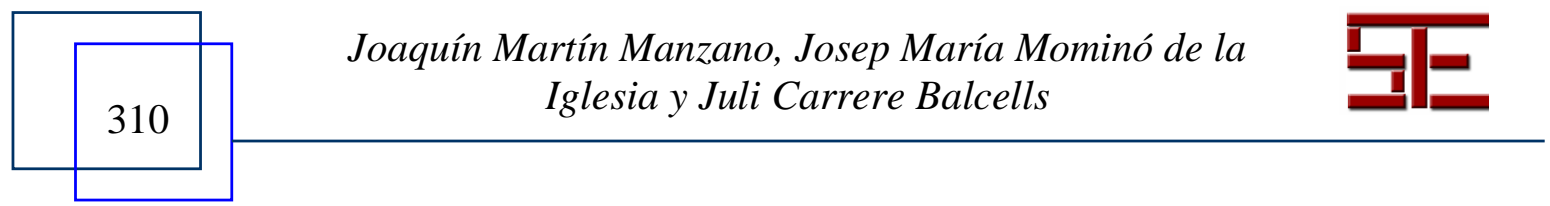




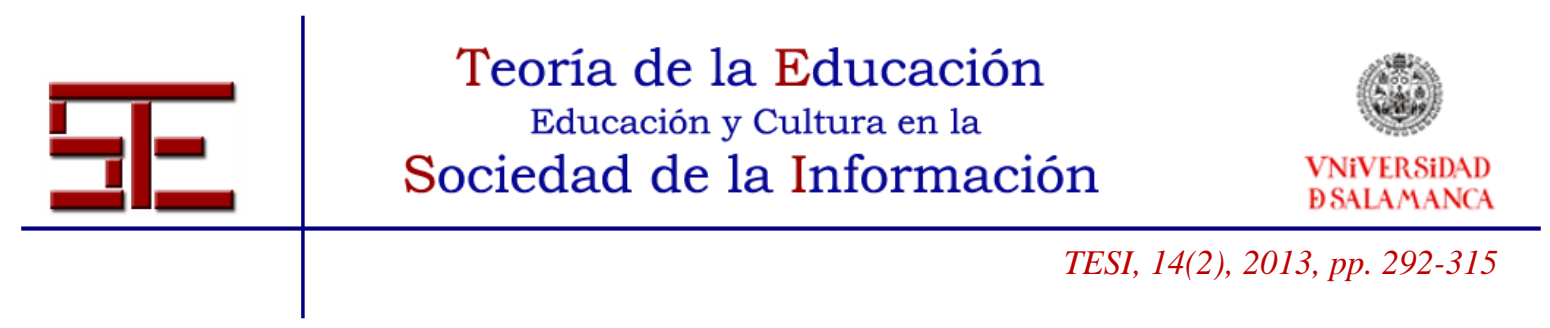

- (2005). Understanding successful school leadership: Progress on a broken front. Journal of Educational Administration, 43, 619-629.

- (2006). Transformational school leadership: Introduction. School effectiveness and school improvement, vol. 17, $\mathrm{n}^{\mathrm{o}} 2$, June, 143-144.

Leithwood. K.; Day, C.; Sammons, P.; Harris, A. \& Hopkins, D. (2006). Seven strong claims about successful school leadership. Nottingham: National College for School Leadership.

Leithwood, K. \& Day, C. (2007). Starting with what we know. En C. Day \& K. Leithwood. K. (Eds.), Successful principal leadership in times of change: An international perspective, 1-16. Dordrecht, The Netherlands: Springer Publishers.

Leithwood, K. A. y Riehl, C. (2003). What do we already know about educational leadership? Philadelphia: Laboratory for Student Success, Temple University.

Levine, D. \& Lezotte, L. (1990). Unusually effective schools: A review of research and practice. Madison, WI: National Center for Effective Schools Research and Development.

LEY GENERAL DE EDUCACIÓN. Ley 14/1970, de 4 de agosto, General de Educación.

LOCE. Ley orgánica 10/2002, de 23 de diciembre, de Calidad de la Educación.

LODE. Ley Orgánica 8/1985, de 3 de julio, Reguladora del Derecho a la Educación.

LOE. Ley Orgánica 2/2006, de 3 de mayo, de Educación.

LOECE. Ley Orgánica 5/1980, de 19 de junio, Reguladora del Estatuto de Centros docentes.

LOGSE. Ley Orgánica 1/1990, de 3 de octubre de 1990, de Ordenación General del Sistema Educativo.

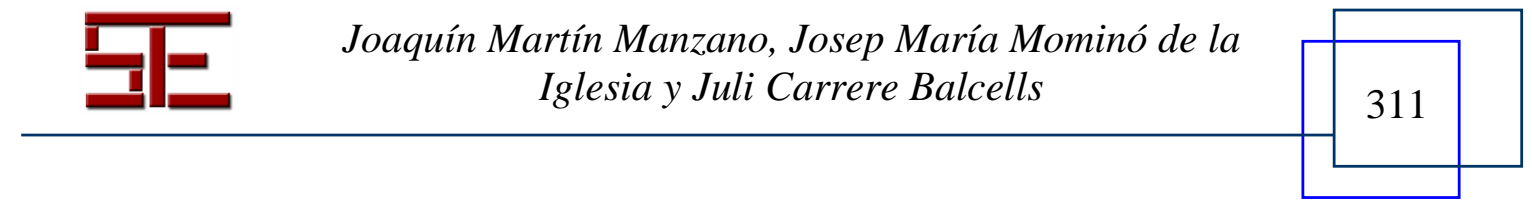




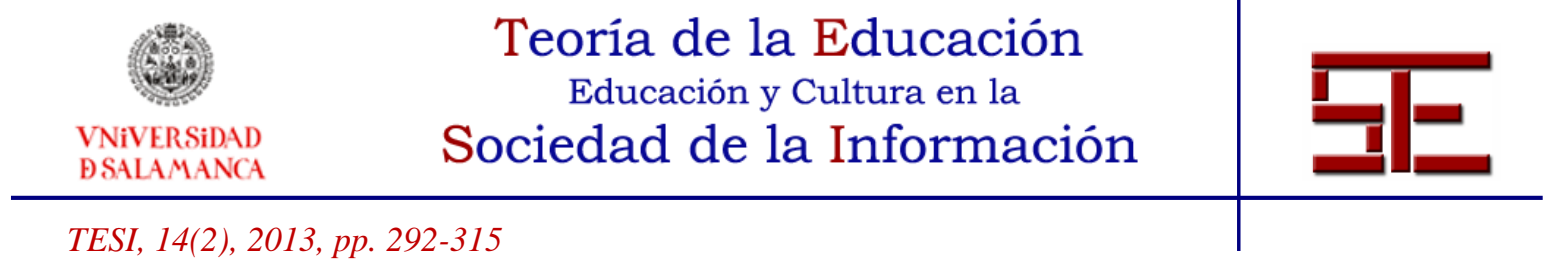

LOMCE. Anteproyecto de ley orgánica para la mejora de la calidad educativa. Extraído el 18 de marzo de 2013 de http://www.mecd.gob.es/servicios-al-ciudadano$\mathrm{mecd} / \mathrm{dms} / \mathrm{mecd} / \mathrm{servicios}-a l-c i u d a d a n o-m e c d / p a r t i c i p a c i o n-p u b l i c a / l o m c e / 20130213$ lomce.pdf.

LOPEG. Ley Orgánica 9/1995, de 20 de noviembre, de la participación, la evaluación y el gobierno de los centros docentes.

Lorenzo, M. (2005). El liderazgo de las organizaciones educativas: revisión y perspectivas actuales. Revista Española de Pedagogía, 232, 367-389.

MacBeath, J. y Dempster, N. (2008). Connecting Leadership and Learning: Principals for practice. London: Rouledge.

MacBeath, J. and Mortimore, P. (2001). Improving School Effectiveness. Buckingham: Open University Press.

McKinsey \& Company (2010). How the world's top school systems are building leadership capacity for the future. New York.

Miles, M. B. y Ekholm, M. (1985). What is School Improvement? En W.G. Van Velzen et al., Making School Improvement Work: A Conceptual Guide to Practice. (pp.33-67). Leuven: ACCO.

Montero, A. (2010a). De la dirección al liderazgo. Los docentes como líderes pedagógicos. Escuela, 3855, 37.

- $\quad$ (2010b). Las responsabilidades del liderazgo escolar. Una dirección para la enseñanza. Escuela, 3871, 39.

Mortimore, P. et al., (1988). The effects of school memership on pupils' educational outcomes. Research Papers in Education, 3, 3-26.

Murillo, F. J. (2005). La investigación sobre eficacia escola. Barcelona: Octaedro.

- (2006). Una Dirección Escolar para el Cambio: del Liderazgo Transformacional al Liderazgo Distribuido. Revista Iberoamericana sobre Calidad, Eficacia y Cambio en Educación, 4, 11-24.

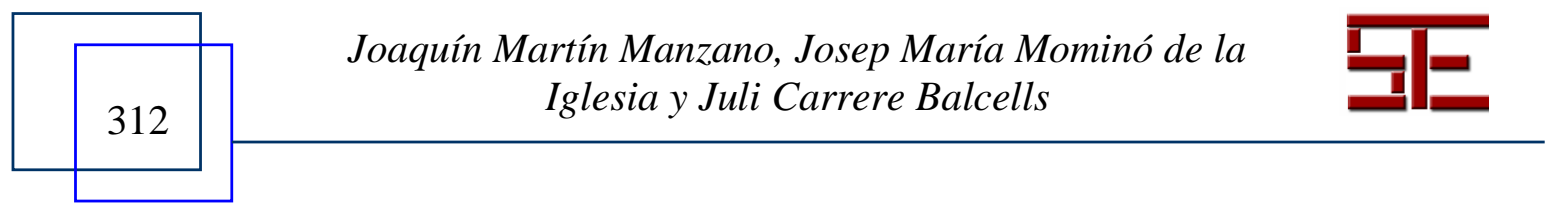




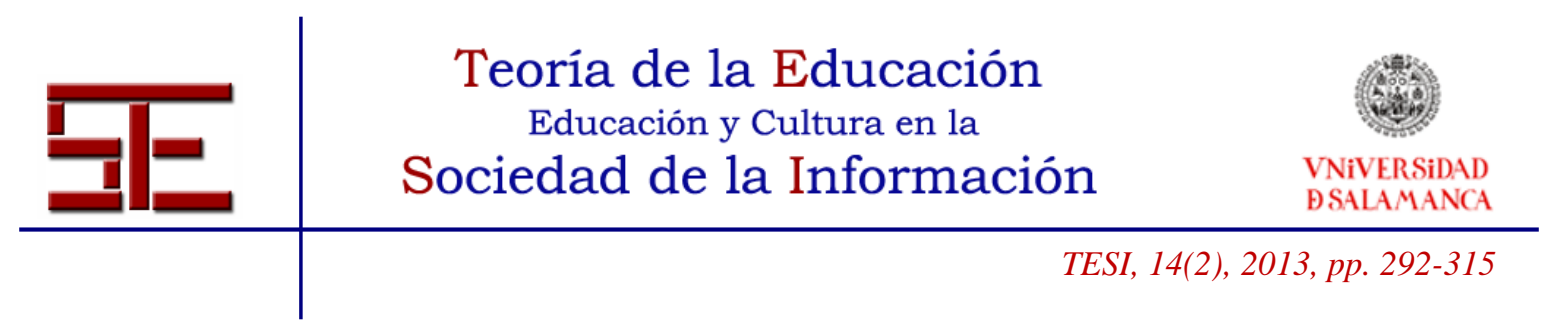

Murphy, J. (1991). Restructuring Schools: Capturing and Assesing the Phenomena. New York. Teachers College Press.

Murphy, J., Elliott, S. N., Goldring, E. \& Porter, A. C. (2006). Learning-centered leadership: A conceptual foundation. New York: The Wallace Foundation.

OCDE (2009). Estudio internacional sobre enseñanza y aprendizaje TALIS. Informe Español 2009. Madrid: Instituto de evaluación. Ministerio de Educación.

OECD (2003). The PISA 2003 assessment framework. Mathematics, reading, science and problem solving knowledge and skills. Paris: OECD.

- (2008). Innovating to Learn, Learning to Innovate. Paris: OECD Publishing.

- (2010). The Nature of Learning: Using Research to Inspire Practice. Paris: OECD Publishing.

OFSTED (2000). Improving City Schools. London: Office for Standards in Education. Pont, B., Nusche, D. \& Moorman, H. (2008). Improving School Leadership. Volume 1: Policy and Practice, OECD Publishing.

Real Decreto 1275/1981. Reglamento de Selección y Nombramiento de Directores.

Reinolds, D. (1976). The delinquent school. En P. Woods (Ed.), The process of schooling. Londres: Routledge and Kegan.

Reynolds et al., (1996). Making Good Schools. London: Routledge.

Reynolds, D. y Creemers, B. (1990). School Effectiveness and School Improvement: A Mission Statement. School Effectiveness \& School Improvement, 1, 1-3.

Robertson, P., Wohlstetter, P. \& Mohrman, S.A. (1995). Generating curriculum and instructional changes through school-based management. Educational Administration Quarterly, 31, 375-404.

Robinson, V. (2007). School Leadership and Student Outcomes: Identifying What Works. Winmalee: Australian Council for Educational Leaders.

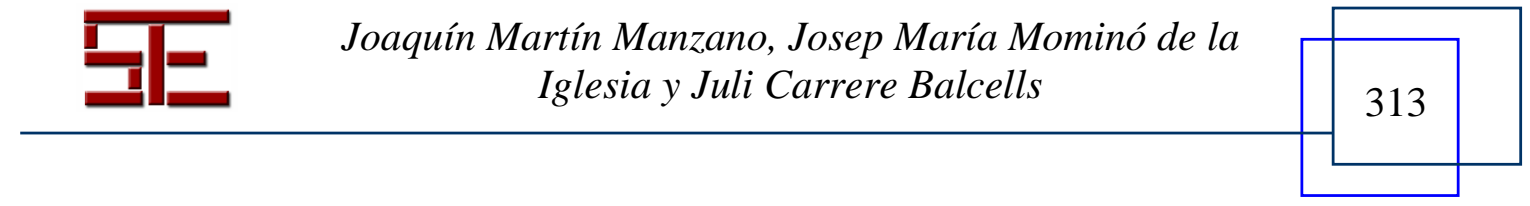




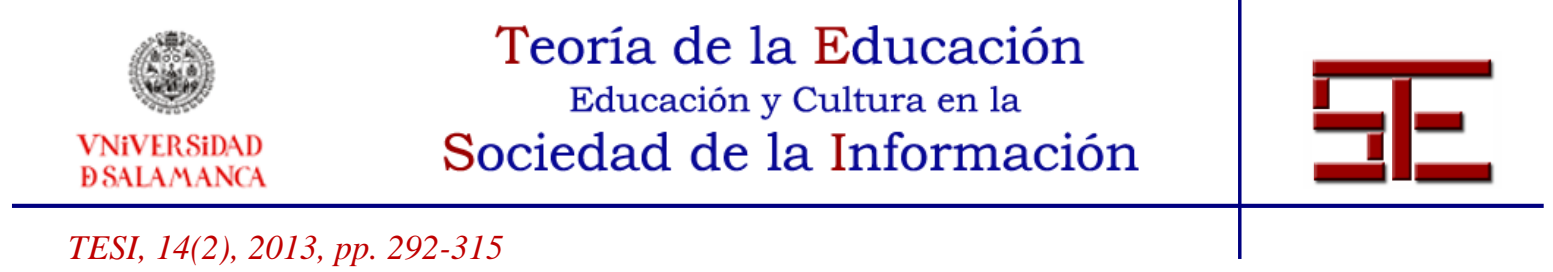

Robinson, V., Hohepa, M. y Lloyd, C. (2009). School Leadership and student outcomes: Identifying what works and why. Best Evidence Synthesis Iteration. Wellington: Ministry of Education.

Rutter, M. (1980). Changing youth in a changing society. Cambridge, MA: Harvard University Press.

Rutter, M., Maughan, B., Mortimore, P. \& Ouston, J. (1979). Fifteen thousand hours. Londres: Open Books.

Sammons, P., Hillman, J. y Mortimore, P. (1995). Key Characteristics of Effective School: A review of School Effectiveness Research. Londres.

Scheerens, J. \& Bosker, R. J. (1997). The foundations of educational effectiveness. Oxford: Elsevier Science Ltd.

Sigalés, C., Mominó, J. M., Meneses, J. y Badia, A. (2008). La Integración de Internet en la Educación Escolar Española: Situación Actual y Perspectivas de Futuro. Barcelona: UOC.

Spillane, J. (2006). Distributed leadership. New York: Jossey-Bass.

Spillane, J. P., Halverson, R. y Diamond, J. B. (2004). Towards a theory of leadership practice: a distributed perspective. Journal of Curriculum Studies, 36, 3-34.

Spillane, J. P. y Coldren, A. F. (2011). Diagnosis an desing for school improvement. New York: Teachers College Press.

Stegö, N. E. et al., (1987). The role of school leaders in school improvement. Leuven: ACCO.

Teddie, C. \& Stringfield, S. (1993). Schools Make a Difference: On Lessons Learned From a 10-Year Study of School Effects. New York: Teachers College Press.

Townsend, T. \& MacBeath, J. (2011). International Handbook on Leadership for Learning. Dordrecht, The Netherlands: Springer.

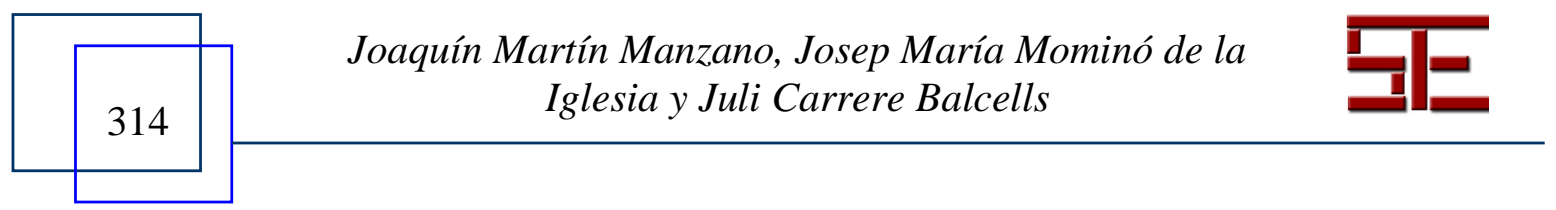




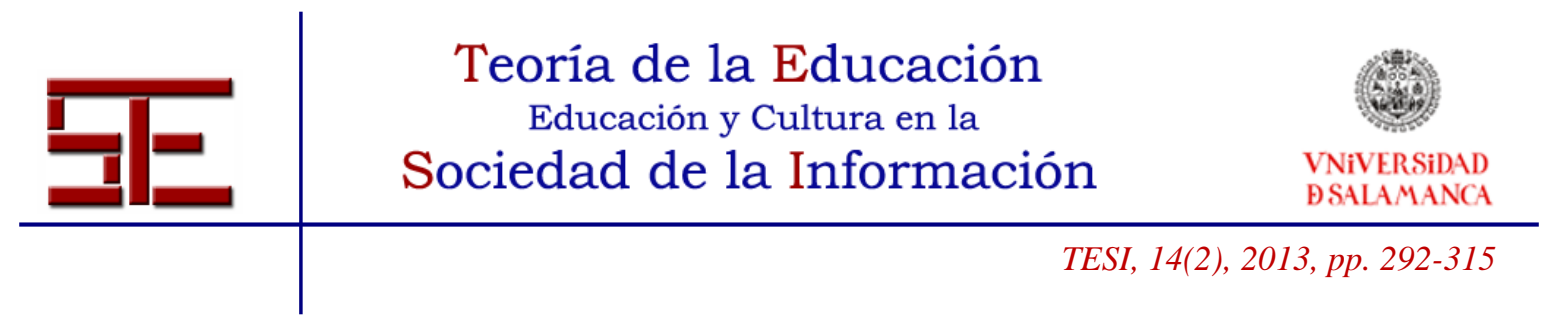

Van Velzen, W. (1979). Autonomy of the School. S'Hertogenkosch: PKC.

Van Velzen, W., Miles, M., Elholm, M., Hameyer, U. \& Robin, D. (1985). Making school improvement work. Leuven, Belgium: ACCO.

Waite, D. \& Nelson, S. (2005). Teaching and Leadership for Social Justice and Social Responsibility: Home is Where the Struggle Starts. Journal of Educational Administration and Foundation, 200-221. Texas State University: San Marcos.

West, M., Jackson, D., Harris, A. y Hopkins, D. (2000). Leadership for School Improvement. En K. Riley \& K. Seashore Louis. Leadership for Change. London: Routledge Falmer Press.

Para citar el presente artículo puede utilizar la siguiente referencia:

Martín Manzano, J., Mominó de la Iglesia, J. M., y Carrere Balcells, J. (2013). La planificación estratégica, un indicador sobre el liderazgo pedagógico. Revista Teoría de la Educación: Educación y Cultura en la Sociedad de la Información. 14(2), 292-315 [Fecha de consulta: dd/mm/aaa].

http://campus.usal.es/ revistas_trabajo/index.php/revistatesi/article/view/10225/10633

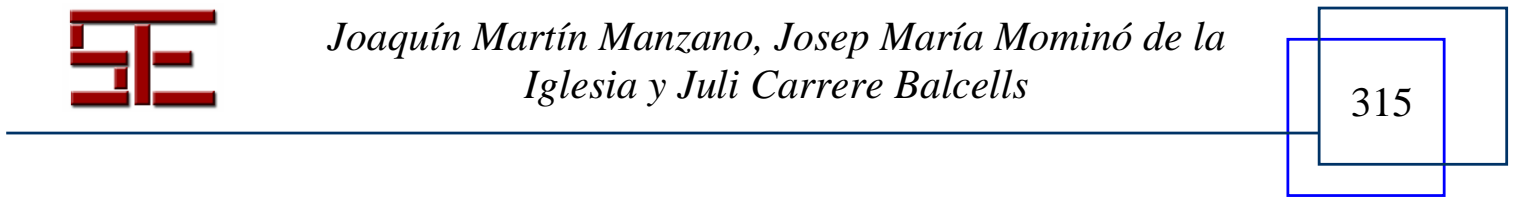

